\title{
Summing Pomeron loops in the dipole approach
}

\section{E. Levin ${ }^{\S *}$ J. Miller ${ }^{\S \dagger}$ and A. Prygarin $\delta ₫$}

\author{
$\S$ Department of Particle Physics, School of Physics and Astronomy \\ Raymond and Beverly Sackler Faculty of Exact Science \\ Tel Aviv University, Tel Aviv, 69978, Israel \\ ${ }^{\top}$ Department of Particle Physics, University of Santiago de Compostela, \\ Santiago de Compostela, 15782, Spain
}

'ABstract: In this paper we argue that in the kinematic range given by $1 \ll \ln \left(1 / \alpha_{S}^{2}\right) \ll \alpha_{S} Y \ll \frac{1}{\alpha_{S}}$, we can reduce the Pomeron calculus to the exchange of non-interacting Pomerons with the renormalized amplitude of their interaction with the target. Therefore, the summation of the Pomeron loops can be performed using the improved Mueller, Patel, Salam and Iancu approximation and this leads to the geometrical scaling solution. This solution is found for the simplified BFKL kernel. We reproduce the findings of Hatta and Mueller that there are overlapping singularities. We suggest a way of dealing with these singularities.

Keywords: BFKL Pomeron, Pomeron loops, Mean field approach, Exact solution.

*leving@post.tau.ac.il, levin@mail.desy.de;

†jeremy@post.tau.ac.il;

†prygarin@post.tau.ac.il

$\S$ permanent address 


\section{Contents}

1. Introduction

2. The BFKL calculus in zero transverse dimension: non-interacting Pomerons and improved Mueller-Patel-Salam-Iancu approach 3

3. Calculation of the simplest diagrams in the BFKL Pomeron calculus 6

3.1 The functional integral formulation of the BFKL Pomeron calculus

3.2 Triple Pomeron vertex in momentum representation 8

3.3 The simplest 'fan' diagram 11

3.3.1 The single Pomeron exchange 11

3.3.2 The first 'fan' diagram 12

3.4 The first enhanced diagram 16

3.5 Reduction of the emhanced diagrams to the system of non-interacting Pomerons 18

4. General solution for the simplified BFKL kernel 20

\begin{tabular}{lll}
4.1 & The main idea & 20 \\
\hline
\end{tabular}

4.2 The simplified BFKL kernel and MFA solution 21

4.3 Improved MPSI solution for Pomeron loops 23

4.4 Self-consistency check 25

$\begin{array}{ll}\text { 5. Conclusions } & 26\end{array}$

\section{Introduction}

In this paper we discuss a solution for the high energy scattering amplitude in the case of high density QCD. We would like to go beyond the mean field approximation, where the solution has been discussed and well understood both analytically and numerically (see [1, 2, 3, 4, 5, 6, 7, 8, ), and to approach the summation of the so called Pomeron loops that should be taken into account 19, 10, 11, 12, 13. The problem of taking into account the Pomeron loops can be reduced to the BFKL Pomeron calculus [14, 15, 16] and/or reduced to the solution of the statistical physics problem, i.e. the Langevin equation and directed percolation [17, 18, 19.

At the moment the problem of the high energy amplitude in QCD is well understood and can be solved only in the kinematic region given by

$$
1 \ll \ln \left(1 / \alpha_{S}^{2}\right) \ll \alpha_{S} Y \ll \frac{1}{\alpha_{S}}
$$


To go beyond this region we need to know the corrections of the order $\alpha_{S}^{2}$ to the BFKL kernel as well as the corrections to the vertices of the Pomeron interaction. Eq. (1.1) will play a significant role in our discussion and we wish to present a more detailed derivation. It is well known that the exchange of one BFKL Pomeron leads to a contribution which can be written as

$$
A \text { (one Pomeron exchange) } \propto \alpha_{S}^{2} e^{\Delta Y}
$$

where $Y=\ln \left(s / s_{0}\right)$ ( $s=W^{2}$ and $W$ is the energy of the scattering in the c.m. frame ). The intercept of the BFKL Pomeron can be represented in the form

$$
\Delta=\alpha_{S} \chi_{\mathrm{LO} B F K L}(\gamma=1 / 2)+\alpha_{S}^{2} \chi_{\mathrm{NLO} \operatorname{BFKL}}(\gamma=1 / 2)
$$

where $\chi$ is the Mellin transform of the BFKL kernel (see more details in [20]).

The contribution from the exchange of two BFKL Pomerons is proportional to

$$
A \text { (two Pomeron exchange) } \propto\left(\alpha_{S}^{2} e^{\Delta Y}\right)^{2}
$$

Comparing Eq. (1.2) to Eq. (1.4) one can see three different kinematic regions:

1. $2 \ln \left(1 / \alpha_{S}\right)>\Delta Y \geq 1$

In this kinematic region we need to take into account the BFKL Pomeron at leading order since $\alpha_{S}^{2} Y \ll 1$ and neglect the contribution from multi Pomeron exchanges.

2. $1 / \alpha_{S} \gg \Delta Y \gg 2 \ln \left(1 / \alpha_{S}\right)$;

We need to take into account multi Pomeron exchange but we can still restrict ourselves to the BFKL kernel in the leading order approximation.

3. $\Delta Y \gg 1 / \alpha_{S} ;$

In this region we have to calculate the next-to-leading BFKL corrections to the BFKL kernel as well as the corrections to the Pomeron vertices. In addition, it is not clear whether or not we can rely on the BFKL Pomeron calculus in this region [21].

Concentrating on the kinematic region of Eq. (1.1) we develop a strategy which consists of three steps. First, we show in this paper that the BFKL Pomeron interaction in this kinematic region can be reduced to the exchange of non-interacting Pomerons if we renormalize the low energy amplitude of the interaction of 'wee' partons with the target.

Using this observation we propose an improved Mueller-Patel-Salam-Iancu method (MPSI) for summation of Pomeron loop diagrams. This method is actually the $t$-channel unitarity constraints re-adjusted in a convenient form for use in the dipole approach of QCD [22, 23].

Finally we propose that the answer which we obtain, is the real solution to our problem at ultra high energy. We will justify and show that this is a reasonable hypothesis.

This paper is organised in the following way. In the next section we discuss the simple case of the BFKL Pomeron calculus in zero transverse dimensions. Being the simplest model for the Pomeron interaction this approach allows us to discuss our main ideas and suggestions without complex calculations. Actually, the main content of this section has been discussed in [24], but for completeness we present this model in our paper. We hope that the reader will find this instructive in later sections. 


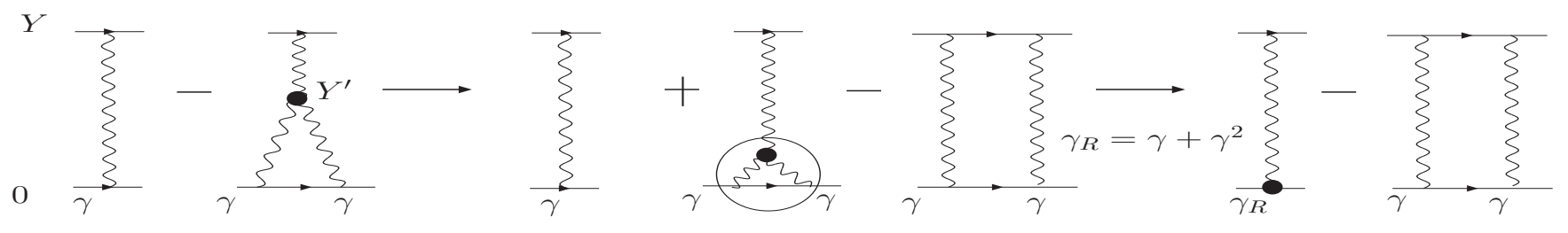

Figure 1: The renormalization procedure in the case of the simplest 'fan' diagram.

In section 3 we argue that the main properties of the BFKL Pomeron calculus in zero transverse dimension are inherent for the BFKL Pomeron calculus in QCD. In particular, we can consider the scattering amplitude in the kinematic region of Eq. (1.1) as the exchange of BFKL Pomerons neglecting their mutual interactions.

Section 4 is devoted to the calculation of the scattering amplitude in the model for the BFKL kernel which has been developed in Ref. [25]. In this section we demonstrate how the MPSI approach works and we obtain a formula for the scattering amplitude. We advocate that the resulting scattering amplitude satisfies the unitarity constraints both in the $s$ and $t$ channels and could be a good candidate for the answer outside the kinematic region given by Eq. (1.1).

In our conclusion we summaries our results and compare them with the approaches that we currently have on the market.

\section{The BFKL calculus in zero transverse dimension: non-interacting Pomerons and improved Mueller-Patel-Salam-Iancu approach}

In this section we analyse the Pomeron diagrams in the BFKL Pomeron calculus in zero transverse dimensions (toy model of [22, 26, 27]). Recently, this model has become rather popular (see [28] and 29] where the most important aspects of this model have been discussed and solved)

Firstly we consider the simplest 'fan' diagram of Fig. 1 and Fig. 2. It can be calculated in an obvious way, using the explicit expression for the Pomeron Green function, namely, $G\left(Y-Y^{\prime}\right)=\exp \left(\Delta\left(Y-Y^{\prime}\right)\right)$. Indeed, for the diagrams of Fig. 1 we have

$$
\begin{aligned}
A(\text { Fig. 囵 }) & =\gamma G(Y-0)-\Delta \gamma^{2} \int_{0}^{Y} d Y^{\prime} G\left(Y-Y^{\prime}\right) G^{2}\left(Y^{\prime}-0\right) \\
& =\gamma e^{\Delta Y}-\Delta \gamma^{2} \int_{0}^{Y} d Y^{\prime} e^{\Delta\left(Y+Y^{\prime}\right)} \\
& =\gamma e^{\Delta Y}-\Delta \gamma^{2}\left(\frac{1}{\Delta} e^{2 \Delta Y}-\frac{1}{\Delta} e^{\Delta Y}\right) \\
& =-\gamma^{2} e^{2 \Delta Y}+\left(\gamma+\gamma^{2}\right) e^{\Delta Y}=-\gamma^{2} e^{2 \Delta Y}+\gamma_{R} e^{\Delta Y}
\end{aligned}
$$

where $\Delta$ is the Pomeron intercept which is equal to the triple Pomeron vertex $\Gamma(1 \rightarrow 2)$ in this oversimplified model, and $\gamma$ is the amplitude of the Pomeron interaction with the target.

For the diagrams of the second order, given by Fig. 2, we have to integrate over the two rapidity variables $y_{1}$ and $y_{2}$. The result is 

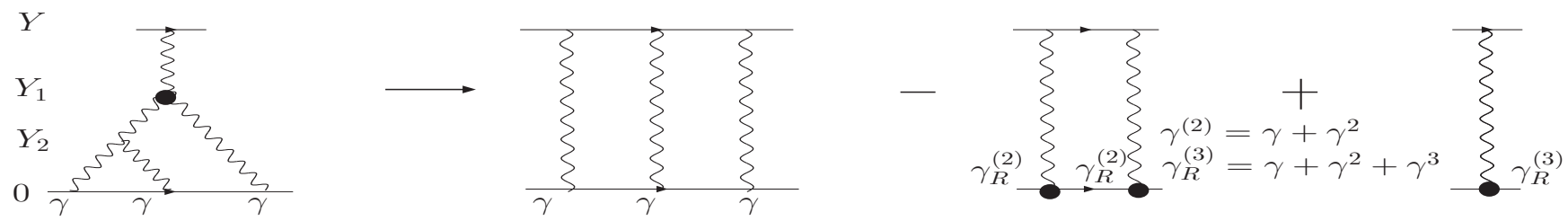

Figure 2: The renormalization procedure in the case of the 'fan' diagram of the second order.

$A($ Fig. 2) $=$

$$
\begin{aligned}
& =2 \Delta^{2} \gamma^{3} \int_{0}^{Y} d y_{1} \int_{0}^{y_{1}} d y_{2} G\left(Y-y_{1}\right) G\left(y_{1}-0\right) G\left(y_{2}-0\right) G^{2}\left(y_{2}-0\right) \\
& =2 \Delta^{2} \gamma^{3} \int_{0}^{Y} d y_{1} \int_{0}^{y_{1}} d y_{2} e^{\Delta\left(Y+y_{1}+y_{2}\right)}=2 \Delta^{2} \gamma^{3}\left(\frac{1}{2 \Delta^{2}} e^{3 \Delta Y}-\frac{1}{\Delta^{2}} e^{2 \Delta Y}+\frac{1}{2 \Delta^{2}} e^{\Delta Y}\right)
\end{aligned}
$$

As one can see, the integration over $Y^{\prime}$ in Fig. 1 and over $y_{1}$ and $y_{2}$ in Fig. 2 reduces these two diagrams to the sum over diagrams which describes two contributions: the exchange of two non-interacting Pomerons and the exchange of one Pomeron with the renormalized vertices: $\gamma_{R}^{(2)}=\gamma+\gamma^{2}$ and $\gamma_{R}^{(3)}=\gamma+\gamma^{2}+\gamma^{3}$

Adding the two contributions of these diagrams gives

$$
\begin{aligned}
& A(\text { Fig. 国 })+A(\text { Fig. 2) }= \\
& =-\gamma^{3} e^{3 \Delta Y}-\left(\gamma^{2}+2 \gamma^{3}\right) e^{2 \Delta Y}+\left(\gamma+\gamma^{2}+\gamma^{3}\right) e^{3 \Delta Y}=\gamma^{3} e^{3 \Delta Y}-\left(\gamma_{R}^{(2)}\right)^{2} e^{2 \Delta Y}+\gamma_{R} e^{\Delta Y}
\end{aligned}
$$

Therefore, one can see that the scattering amplitude can be rewritten as the exchange of Pomerons without interaction between them but with the renormalized Pomeron - particle vertex. In the dipole model this vertex is the amplitude of the two dipole interaction in the Born approximation of perturbative QCD.

These two examples illustrate our main idea: the BFKL Pomeron calculus in zero transverse dimensions can be viewed as the theory of free, non-interacting Pomerons whose interaction with the target has to be renormalized. The master equation for the scattering amplitude in the mean field approximation can be easily rewritten (see Ref. [24]) in the form

$$
\frac{\partial N_{0}\left(\gamma_{R} \mid Y\right)}{\partial Y}=\Gamma(1 \rightarrow 2) \gamma_{R} \frac{\partial N_{0}\left(\gamma_{R} \mid Y\right)}{\partial \gamma_{R}}
$$

with

$$
\gamma_{R}=\frac{\gamma}{1-\gamma}
$$

Thus we have shown in Eq. (2.1) and Eq. 2.3) how Eq. 2.6 has started to build up in the perturbative expansion.

The general solution of Eq. (2.5) is the system of non - interacting Pomerons and the scattering amplitude can be found in the form

$$
N_{0}\left(\gamma_{R} \mid Y\right)=\sum_{n=1}^{\infty}(-1)^{n} C_{n} \gamma_{R}^{n} G^{n}(Y-0)
$$

where the coefficients $C_{n}$ could be found from the initial conditions, namely, from the expression for the low energy amplitude. In particular, the initial condition

$$
N_{0}\left(\gamma_{R} \mid Y=0\right)=\gamma=\gamma_{R} /\left(1+\gamma_{R}\right)
$$


generates $C_{n}=1$ and the solution is

$$
N_{0}\left(\gamma_{R} \mid Y\right)=\frac{\gamma_{R} e^{\Delta Y}}{1+\gamma_{R} e^{\Delta Y}}
$$

The initial condition of Eq. (2.8) has very simple physics behind it that has been discussed in Ref. 24.

For the analysis of the enhanced diagrams we start from the first diagram of Fig. 3, which can be written as follows

$$
\begin{aligned}
& A(\text { Fig. B) }= \\
& -\Delta^{2} \gamma^{2} \int_{0}^{Y} d y_{1} \int_{0}^{y_{1}} d y_{2} G\left(Y-y_{1}\right) G^{2}\left(y_{1}-y_{2}\right) G\left(y_{2}-0\right) \\
& =-\Delta^{2} \gamma^{2} \int_{0}^{Y} d y_{1} \int_{0}^{y_{1}} d y_{2} e^{\Delta\left(Y+y_{1}-y_{2}\right)} \\
& =-\gamma^{2} e^{2 \Delta}+\gamma^{2} e^{\Delta Y}+\Delta \gamma^{2} Y e^{\Delta Y}
\end{aligned}
$$

where $\Gamma(2 \rightarrow 1)=\Delta \gamma^{2}$ (see Ref.[1]).

Adding Eq. (2.10) to the exchange of one Pomeron we obtain the following

$$
\text { One Pomeron exchange }+A(\text { Fig. B })=\gamma_{R} e^{\Delta_{R} Y}-\gamma^{2} e^{2 \Delta Y}
$$

with

$$
\gamma_{R}=\gamma^{(2)}=\gamma+\gamma^{2} ; \quad \Delta_{R}=\Delta+\gamma \Delta ;
$$

Therefore, the Pomeron loops can be either large ( where their size in rapidity is of the order of $Y$ ) and they can be considered as un-enhanced diagrams; or small (where their sizes are of the order $1 / \Delta$ ) and they can be treated as the renormalisation of the Pomeron intercept.

In QCD $\Delta \propto \bar{\alpha}_{S}$ while $\gamma \propto \alpha_{S}^{2}$. Therefore, the renormalisation of the Pomeron intercept $\Delta$ is proportional to $\alpha_{S}^{3}$. We can neglect this contribution since (i) there a lot of $\alpha_{S}^{2}$ corrections to the kernel of the BFKL equation that are much larger than this contribution; and (ii) in the region of $Y \ll 1 / \alpha_{S}^{2}$, where we can trust our Pomeron calculus (see introduction) $\left(\Delta_{R}-\Delta\right) Y \ll 1$.

Concluding this analysis we can claim that the BFKL Pomeron calculus in zero dimension is a theory of noninteracting Pomerons with renormalised vertices of the Pomeron-particle interaction. In the dipole language, it means that we have a system non-interacting Pomerons with a specific hypothesis on the amplitude of the dipole interactions at low energy. For the problem that we are solving here, namely, when we have one bare Pomeron at low energy, this amplitude is determined by Eq. 2.8.).

For such a system we can calculate the scattering amplitude using a method suggested by Mueller, Patel, Salam and Iancu and developed in a number of papers (see Refs. 23, 36, 32, 37, 35, 38, and references therein). In this method the scattering amplitude is calculated using the $t$-channel unitarity constraints which is written in the following way (assuming that the amplitudes at high energy are purely imaginary, $N=\operatorname{Im} A$ ):

$$
N([\ldots] \mid Y)=N\left([\ldots] \mid Y-Y^{\prime} ; P \rightarrow n P\right) \bigotimes N\left([\ldots] \mid Y^{\prime} ; P \rightarrow n P\right)
$$

where $\bigotimes$ stands for all necessary integrations while [...] describes all quantum numbers (dipole sizes and so on ).

The correct implementation of Eq. (2.13) leads for our case to the following formula (see also Refs. 35, 36, 30, )

$$
N_{0}^{M P S I}(Y)=1-\exp \left\{-\gamma^{B A} \frac{\partial}{\partial \gamma_{R}^{(1)}} \frac{\partial}{\partial \gamma_{R}^{(2)}}\right\} N^{M F A}\left(( \gamma _ { R } ^ { ( 1 ) } | Y - Y ^ { \prime } ) N ^ { M F A } \left(\left.\left(\gamma_{R}^{(2)} \mid Y^{\prime}\right)\right|_{\gamma_{R}^{(1)}}=\gamma_{R}^{(2)}=0\right.\right.
$$


where $N^{M F A}\left(Y, \gamma_{R}\right)$ is given by Eq. 2.9) (see also Eq. 2.5)) in the mean field approximation and $\gamma^{B A} \propto \alpha_{S}^{2}$ is the scattering amplitude at low energies which is described by the Born approximation in perturbative QCD. The difference between Eq. (2.14) and the original MPSI approach is the fact that this equation does not depend on the value of $Y^{\prime}$ and, because of this, we do not need to choose $Y^{\prime}=Y / 2$ for the best accuracy.

Substituting Eq. (2.9) in Eq. (2.14) we obtain

$$
N_{0}^{M P S I}\left(\gamma^{B A} \mid Y\right)=1-\exp \left(\frac{1}{\gamma^{B A} e^{\Gamma(1 \rightarrow 2) Y}}\right) \frac{1}{\gamma^{B A} e^{\Gamma(1 \rightarrow 2) Y}} \Gamma\left(0, \frac{1}{\gamma^{B A} e^{\Gamma(1 \rightarrow 2) Y}}\right)
$$

$\Gamma(0, x)$ is the incomplete gamma function (see formulae $\mathbf{8 . 3 5 0} \mathbf{- 8 . 3 5 9}$ in Ref. [40]).

We claim that Eq. (2.15) is the solution to our problem. One can easily see that $N_{0}(\gamma \mid Y) \rightarrow 1$ at high energies in contrast to the exact solution (see [30]). The exact solution leads to the amplitude that vanishes at high energy (see Refs. 29, 39). As has been mentioned the solution depends crucially on the initial condition for the scattering amplitude at low energies. For Eq. (2.15) this amplitude is equal to

$$
N_{0}^{M P S I}(\gamma \mid Y=0)=\sum_{n=1}^{\infty}(-1)^{n+1} n !\left(\gamma^{B A}\right)^{n}
$$

with $\gamma^{B A} \propto \alpha_{S}^{2}$.

Eq. (2.14) can be rewritten in a more convenient form using the Cauchy formula for the derivatives, namely,

$$
\frac{\partial^{n} Z^{M F A}\left(\gamma_{R} \mid Y\right)}{\partial \gamma_{R}^{n}}=n ! \frac{1}{2 \pi i} \oint_{C} \frac{Z^{M F A}\left(\gamma_{R}^{\prime} \mid Y\right)}{\gamma_{R}^{\prime n+1}} d \gamma_{R}^{\prime}
$$

The contour $C$ in Eq. (2.17) is a circle with a small radius around $\gamma_{R}=0$. However, since the function $Z$ does not grow at large $\gamma_{R}$ for $n \leq 1$ we can close our contour $C$ on the singularities of the function $Z$. We will call this new contour $C_{R}$.

$$
\begin{aligned}
N_{0}^{M P S I}(Y) & =1-\exp \left\{-\gamma^{B A} \frac{\partial}{\partial \gamma_{R}^{(1)}} \frac{\partial}{\partial \gamma_{R}^{(2)}}\right\} N^{M F A}\left(( \gamma _ { R } ^ { ( 1 ) } | Y - Y ^ { \prime } ) N ^ { M F A } \left(\left.\left(\gamma_{R}^{(2)} \mid Y^{\prime}\right)\right|_{\gamma_{R}^{(1)}=\gamma_{R}^{(2)}=0}\right.\right. \\
& =1-\sum_{n=1}^{\infty} \frac{\left(-\gamma^{B A}\right)^{n}}{n !} n ! n ! \frac{1}{(2 \pi i)^{2}} \oint_{C_{R}^{1}} d \gamma_{R}^{(1)} \frac{Z^{M F A}\left(\gamma_{R}^{(1)} \mid Y-Y^{\prime}\right)}{\left(\gamma_{R}^{(1)}\right)^{n+1}} \oint_{C_{R}^{2}} d \gamma_{R}^{(2)} \frac{Z^{M F A}\left(\gamma_{R}^{(2)} \mid Y^{\prime}\right)}{\left(\gamma_{R}^{(2)}\right)^{n+1}}= \\
& =\frac{1}{(2 \pi i)^{2}} \oint \oint \frac{d \tilde{\gamma}_{R}^{(1)}}{\tilde{\gamma}_{R}^{(1)}} \frac{d \tilde{\gamma}_{R}^{(2)}}{\tilde{\gamma}_{R}^{(2)}}\left\{1-\exp \left(\frac{\tilde{\gamma}_{R}^{(1)} \tilde{\gamma}_{R}^{(2)}}{\gamma^{B A} e^{\mathcal{Y}}}\right) \frac{\tilde{\gamma}_{R}^{(1)} \tilde{\gamma}_{R}^{(1)}}{\gamma^{B A} e^{\mathcal{Y}}} \Gamma\left(0, \frac{\tilde{\gamma}_{R}^{(1)} \tilde{\gamma}_{R}^{(2)}}{\gamma^{B A} e^{\mathcal{Y}}}\right)\right\} \times \\
& \times Z^{M F A}\left(\tilde{\gamma}_{R}^{(1)}\right) Z^{M F A}\left(\tilde{\gamma}_{R}^{(2)}\right)
\end{aligned}
$$

Here we introduce new variables $\tilde{\gamma}_{R}^{(1)}=\gamma_{R}^{(1)} \exp \left(\mathcal{Y}-\mathcal{Y}^{\prime}\right)$ and $\tilde{\gamma}_{R}^{(2)}=\gamma_{R}^{(2)} \exp \left(\mathcal{Y}^{\prime}\right)$. In these new variables

$$
Z^{M F A}\left(\tilde{\gamma}_{R}^{(1)}\right)=\frac{1}{1+\tilde{\gamma}_{R}^{(1)}} ; \quad Z^{M F A}\left(\tilde{\gamma}_{R}^{(2)}\right)=\frac{1}{1+\tilde{\gamma}_{R}^{(2)}}
$$

Closing the integration on the poles $\tilde{\gamma}_{R}^{(1)}=-1$ and $\tilde{\gamma}_{R}^{(2)}=-1$ we obtain the formula of Eq. (2.15).

\section{Calculation of the simplest diagrams in the BFKL Pomeron calculus}

The main goal of this paper is to show that in the general case of the BFKL Pomeron calculus we have the same situation as in the simple model. We start from the analysis of the simple diagrams of the BFKL Pomeron interaction. 


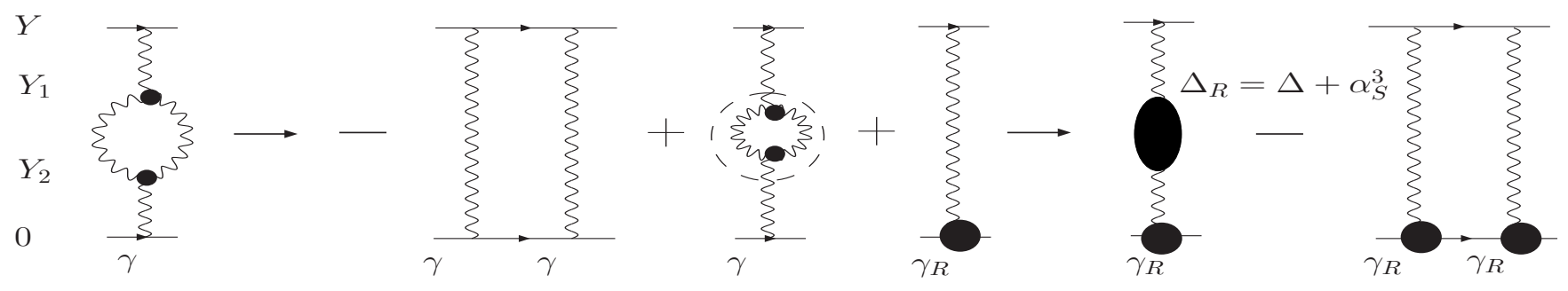

Figure 3: The renormalization procedure in the case of the simplest enhanced diagram.

\subsection{The functional integral formulation of the BFKL Pomeron calculus}

The most economic form of the BFKL Pomeron calculus was suggested and developed by Braun in Ref. 16. where he formulated this theory as the functional integral

$$
Z\left[\Phi, \Phi^{+}\right]=\int D \Phi D \Phi^{+} e^{S} \text { with } S=S_{0}+S_{I}+S_{E}
$$

where $S_{0}$ describes free Pomerons, $S_{I}$ corresponds to their mutual interaction while $S_{E}$ relates to the interaction with external sources (target and projectile).

They have the form

$$
\begin{aligned}
S_{0} & =\int d Y d Y^{\prime} d^{2} \underline{x}_{1} d^{2} \underline{x}_{2} d^{2} \underline{x}_{1}^{\prime} d^{2} \underline{x}_{2}^{\prime} \Phi^{+}\left(\underline{x}_{1}, \underline{x}_{2} ; Y\right) G^{-1}\left(\underline{x}_{1}, \underline{x}_{2} ; Y \mid \underline{x}_{1}^{\prime}, \underline{x}_{2}^{\prime} ; Y^{\prime}\right) \Phi\left(\underline{x}_{1}^{\prime}, \underline{x}_{2}^{\prime} ; Y^{\prime}\right) ; \\
S_{I} & =\frac{2 \pi \bar{\alpha}_{S}^{2}}{N_{c}} \int d Y \int \frac{d^{2} \underline{x}_{1} d^{2} \underline{x}_{2} d^{2} \underline{x}_{3}}{\underline{x}_{12}^{2} \underline{x}_{23}^{2} \underline{x}_{31}^{2}} \cdot\left\{\left(L_{1,2} \Phi\left(\underline{x}_{1}, \underline{x}_{2} ; Y\right)\right) \Phi^{+}\left(\underline{x}_{2}, \underline{x}_{3} ; Y\right) \Phi^{+}\left(\underline{x}_{3}, \underline{x}_{1} ; Y\right)+\text { h.c. }\right\} ; \\
S_{E} & =-\int d Y d^{2} \underline{x}_{1} d^{2} \underline{x}_{2}\left\{\Phi\left(\underline{x}_{1}, \underline{x}_{2} ; Y\right) \tau_{p r}\left(\underline{x}_{1}, \underline{x}_{2} ; Y\right)+\Phi^{+}\left(\underline{x}_{1}, \underline{x}_{2} ; Y\right) \tau_{\text {tar }}\left(\underline{x}_{1}, \underline{x}_{2} ; Y\right)\right\}
\end{aligned}
$$

It should be stressed that Eq. (3.4) describes the local interaction both in rapidity and in coordinates and $\tau_{p r}\left(\tau_{t a r}\right)$ stands for the projectile and target, respectively.

The Pomeron Green function has the form 442, 43]

$$
\begin{aligned}
& G\left(\underline{x}_{1}, \underline{x}_{2} ; Y \mid \underline{x}_{1}^{\prime}, \underline{x}_{2}^{\prime} ; Y^{\prime}\right) \equiv \\
& \theta\left(Y-Y^{\prime}\right) \sum_{n=-\infty}^{\infty} \int d \nu d^{2} \underline{x}_{0} e^{\omega(n, \nu)\left(Y-Y^{\prime}\right)}\left(\nu^{2}+\frac{n^{2}}{4}\right) \lambda(n, \nu) E^{*}\left(\underline{x}_{1}^{\prime}, \underline{x}_{2}^{\prime} ; x_{0} \mid \nu\right) E\left(\underline{x}_{1}, \underline{x}_{2} ; \underline{x}_{0} \mid \nu\right)
\end{aligned}
$$

where the vertices $\mathrm{E}$ are given by

$$
E\left(\underline{x}_{1}, \underline{x}_{2} ; \underline{x}_{0} \mid n ; \nu\right)=(-1)^{n}\left(\frac{x_{12}}{x_{10} x_{20}}\right)^{h}\left(\frac{x_{12}^{*}}{x_{10}^{*} x_{20}^{*}}\right)^{\tilde{h}}
$$

with $x_{i j}=x_{i}-x_{j}, x_{i}=x_{i, x}+i x_{i, y}{ }^{1}, x_{i}^{*}=x_{i, x}-i x_{i, y} ; h=(1+n) / 2+i \nu$ and $\tilde{h}=1-h^{*}=(1-n) / 2+i \nu$.

\footnotetext{
${ }^{1} x_{i, x}$ and $x_{i, y}$ are $\mathrm{x}$ - and y-components of the two dimensional vector $\underline{x}_{i}$
} 
The energy levels $\omega(n, \nu)$ are the BFKL eigenvalues

$$
\omega(n, \nu)=\bar{\alpha}_{S}\left(\psi(1)-\operatorname{Re} \psi\left(\frac{|n|+1}{2}+i \nu\right)\right)
$$

with $\psi(z)=d \ln \Gamma(z) / d z$ and $\Gamma(z)$ is the Euler gamma function.

The operator $L_{12}$ in Eq. (3.1) is defined as

$$
L_{1,2}=\underline{x}_{12}^{4} \underline{p}_{1}^{2} \underline{p}_{2}^{2} \text { with } \underline{p}^{2}=-\nabla^{2}
$$

with $E\left(\underline{x}_{1}, \underline{x}_{2} ; \underline{x}_{0} \mid \nu\right)$ and $\frac{1}{\lambda(n, \nu)}$ being its eigenfunctions and eigenvalues respectively. It is easy to check that

$$
L_{1,2} E\left(\underline{x}_{1}, \underline{x}_{2} ; \underline{x}_{0} \mid n ; \nu\right)=\frac{1}{\lambda(n, \nu)} E\left(\underline{x}_{1}, \underline{x}_{2} ; \underline{x}_{0} \mid n ; \nu\right)
$$

with

$$
\lambda(n, \nu)=\frac{1}{\left[\frac{(n+1)^{2}}{4}+\nu^{2}\right]\left[\frac{(n-1)^{2}}{4}+\nu^{2}\right]}
$$

For our further discussions we rewrite Eq. (3.6) in a more convenient form as

$$
\begin{aligned}
E\left(\underline{x}_{1}, \underline{x}_{2} ; \underline{x}_{0} \mid n ; \nu\right) & \equiv E\left(z_{12}-\frac{x_{12}}{2}, z_{12}+\frac{x_{12}}{2} \mid n ; \nu\right) \\
& =(-1)^{n}\left(\frac{x_{12}}{\left(z_{12}-\frac{x_{12}}{2}\right)\left(z_{12}+\frac{x_{12}}{2}\right)}\right)^{h}\left(\frac{x_{12}^{*}}{\left(z_{12}^{*}-\frac{x_{12}^{*}}{2}\right)\left(z_{12}^{*}+\frac{x_{12}^{*}}{2}\right)}\right)^{\tilde{h}}
\end{aligned}
$$

where the new variable $z_{12}$ is defined by

$$
z_{12}=\frac{x_{1}+x_{2}}{2}-x_{0}
$$

\subsection{Triple Pomeron vertex in momentum representation}

In this section we calculate the triple BFKL Pomeron vertex which is the key ingredient of all enhanced diagrams of the type illustrated in Fig. 3. We find it easier to do all the calculations in the momentum representation. We define the Fourier transform of the vertex function in Eq. (3.6) as

$$
g(\underline{k}, \underline{q}, n, \nu) \equiv \int \frac{d^{2} \underline{x}_{12}}{\underline{x}_{12}^{2}} d^{2} \underline{z} e^{i \underline{k} \cdot \underline{x}_{12}} e^{i q \underline{z}} \underline{z} E\left(\underline{x}_{1}, \underline{x}_{2} ; \underline{x}_{0} \mid n ; \nu\right)
$$

with $\underline{x}_{12}=\underline{x}_{1}-\underline{x}_{2}$ and $\underline{z}=\left(\underline{x}_{1}+\underline{x}_{2}\right) / 2-\underline{x}_{0}=\underline{b}-\underline{x}_{0}$. As it follows from Eq. (3.13), the momentum $\underline{k}$ is defined as the momentum conjugate to the dipole size. It will be shown later that the physical meaning of the momentum $\underline{q}$ is a momentum transferred along the Pomeron. The explicit expression for the function $g(\underline{k}, \underline{q}, n, \nu)$ is found in the Appendix A.

Using the definition of Eq. (3.13) we want to express the free Pomeron Green function defined in Eq. (3.5) in terms of the function $g(\underline{k}, \underline{q}, n, \nu)$. To do this we find an inverse Fourier transform of $g(\underline{k}, \underline{q}, n, \nu)$ and substitute it into Eq. (3.5). The vertex function $E$ in terms of $g(\underline{k}, \underline{q}, n, \nu)$ reads

$$
E\left(\underline{x}_{1}, \underline{x}_{2} ; \underline{x}_{0} \mid n ; \nu\right)=\underline{x}_{12}^{2} \int \frac{d^{2} \underline{k}}{(2 \pi)^{2}} \frac{d^{2} \underline{q}}{(2 \pi)^{2}} e^{-i \underline{k} \cdot \underline{x}_{12}} e^{-i \underline{q} \cdot \underline{z}} g(\underline{k}, \underline{q}, n, \nu)
$$


With the help of Eq. (3.14) we rewrite the free Pomeron Green function in Eq. (3.5) as

$$
\begin{aligned}
& G\left(\underline{x}_{1}, \underline{x}_{2} ; Y \mid \underline{x}_{1}^{\prime}, \underline{x}_{2}^{\prime} ; Y^{\prime}\right) \equiv \\
& \theta\left(Y-Y^{\prime}\right) \sum_{n=-\infty}^{\infty} \int d \nu d^{2} \underline{x}_{0} e^{\omega(n, \nu)\left(Y-Y^{\prime}\right)}\left(\nu^{2}+\frac{n^{2}}{4}\right) \lambda(n, \nu) \times \\
& \underline{x}_{12}^{\prime 2} \int \frac{d^{2} \underline{k^{\prime}}}{(2 \pi)^{2}} \frac{d^{2} \underline{q}^{\prime}}{(2 \pi)^{2}} e^{+i \underline{k}^{\prime} \cdot \underline{x}_{12}^{\prime}} e^{+i \underline{q}^{\prime} \cdot \underline{\prime}_{12}^{\prime}} g^{*}\left(\underline{k}^{\prime}, \underline{q}^{\prime}, n, \nu\right) \underline{x}_{12}^{2} \int \frac{d^{2} \underline{k}}{(2 \pi)^{2}} \frac{d^{2} \underline{q}}{(2 \pi)^{2}} e^{-i \underline{k} \underline{x}_{12}} e^{-i \underline{q} \cdot \underline{z}_{12}} g(\underline{k}, \underline{q}, n, \nu)= \\
& \theta\left(Y-Y^{\prime}\right) \sum_{n=-\infty}^{\infty} \int d \nu d^{2} \underline{x}_{0} e^{\omega(n, \nu)\left(Y-Y^{\prime}\right)}\left(\nu^{2}+\frac{n^{2}}{4}\right) \lambda(n, \nu) \times \\
& \underline{x}_{12}^{\prime 2} \int \frac{d^{2} \underline{k}^{\prime}}{(2 \pi)^{2}} \frac{d^{2} \underline{q}^{\prime}}{(2 \pi)^{2}} e^{+i \underline{k}^{\prime} \cdot \underline{x}_{12}^{\prime}} e^{+i \underline{q}^{\prime} \cdot \underline{b}_{12}^{\prime}} e^{-i \underline{q}^{\prime} \cdot \underline{x}_{0}} g^{*}\left(\underline{k}^{\prime}, \underline{q}^{\prime}, n, \nu\right) \underline{x}_{12}^{2} \int \frac{d^{2} \underline{k}}{(2 \pi)^{2}} \frac{d^{2} \underline{q}}{(2 \pi)^{2}} e^{-i \underline{k} \cdot \underline{x}_{12}} e^{-i \underline{q} \cdot \underline{b}_{12}} e^{+i \underline{q} \cdot \underline{x}_{0}} g(\underline{k}, \underline{q}, n, \nu)
\end{aligned}
$$

The integration over $\underline{x}_{0}$ brings in a delta function $(2 \pi)^{2} \delta^{(2)}\left(\underline{q}-\underline{q}^{\prime}\right)$. After the integration over the momentum $\underline{q}^{\prime}$ we end up with the expression for free Pomeron Green function in terms of $g(\underline{k}, \underline{q}, n, \nu)$ as follows.

$$
\begin{aligned}
& G\left(\underline{x}_{1}, \underline{x}_{2} ; Y \mid \underline{x}_{1}^{\prime}, \underline{x}_{2}^{\prime} ; Y^{\prime}\right) \equiv \\
& \theta\left(Y-Y^{\prime}\right) \sum_{n=-\infty}^{\infty} \int d \nu e^{\omega(n, \nu)\left(Y-Y^{\prime}\right)}\left(\nu^{2}+\frac{n^{2}}{4}\right) \lambda(n, \nu)(2 \pi)^{2} \delta^{2}\left(\underline{q}^{\prime}-\underline{q}\right) \times \\
& \underline{x}_{12}^{\prime 2} \int \frac{d^{2} \underline{k}^{\prime}}{(2 \pi)^{2}} \frac{d^{2} \underline{q}^{\prime}}{(2 \pi)^{2}} e^{+i \underline{k}^{\prime} \cdot \underline{x}_{12}^{\prime}} e^{+i \underline{q}^{\prime} \cdot \underline{b}_{12}^{\prime}} g^{*}\left(\underline{k}^{\prime}, \underline{q}^{\prime}, n, \nu\right) \underline{x}_{12}^{2} \int \frac{d^{2} \underline{k}}{(2 \pi)^{2}} \frac{d^{2} \underline{q}}{(2 \pi)^{2}} e^{-i \underline{k} \cdot \underline{x}_{12}} e^{-i \underline{q} \cdot \underline{b_{12}}} g(\underline{k}, \underline{q}, n, \nu)= \\
& \theta\left(Y-Y^{\prime}\right) \sum_{n=-\infty}^{\infty} \int d \nu e^{\omega(n, \nu)\left(Y-Y^{\prime}\right)}\left(\nu^{2}+\frac{n^{2}}{4}\right) \lambda(n, \nu) \times \\
& \underline{x}_{12}^{\prime 2} \underline{x}_{12}^{2} \int \frac{d^{2} \underline{k}^{\prime}}{(2 \pi)^{2}} \frac{d^{2} \underline{k}}{(2 \pi)^{2}} \frac{d^{2} \underline{q}}{(2 \pi)^{2}} e^{+i \underline{k}^{\prime} \cdot \underline{x}_{12}^{\prime}} e^{-i \underline{k} \cdot \underline{x}_{12}} e^{\left.-i \underline{q} \cdot \underline{b}_{12}-\underline{b}_{12}^{\prime}\right)} g^{*}\left(\underline{k}^{\prime}, \underline{q}, n, \nu\right) g(\underline{k}, \underline{q}, n, \nu)
\end{aligned}
$$

The expression in Eq. (3.16) clarifies the meaning of the momentum $q$ defined in Eq. (3.13) as the momentum conjugate to the difference in the impact parameters of the interacting dipoles or, alternatively, as the momentum transferred along the Pomeron.

Using the same standard procedure we want to calculate the triple Pomeron vertex defined in the BFKL Pomeron Calculus by Eq. (3.3). It is better to write the vertex in terms of free Pomeron Green functions as

$$
\frac{2 \pi \bar{\alpha}_{S}^{2}}{N_{c}} \int d Y \int \frac{d^{2} \underline{x}_{1} d^{2} \underline{x}_{2} d^{2} \underline{x}_{3}}{\underline{x}_{12}^{2} \underline{x}_{23}^{2} \underline{x}_{31}^{2}}\left(L_{12} G\left(\underline{x}_{1}^{\prime}, \underline{x}_{2}^{\prime} ; Y_{1} \mid \underline{x}_{1}, \underline{x}_{2} ; Y\right)\right) G\left(\underline{x}_{2}, \underline{x}_{3} ; Y \mid \underline{x}_{2}^{\prime}, \underline{x}_{3}^{\prime} ; Y_{2}\right) G\left(\underline{x}_{3}, \underline{x}_{1} ; Y \mid \underline{x}_{3}^{\prime}, \underline{x}_{1}^{\prime} ; Y_{3}\right)
$$

and then use the representation of $G\left(\underline{x}_{1}^{\prime}, \underline{x}_{2}^{\prime} ; Y^{\prime} \mid \underline{x}_{1}, \underline{x}_{2} ; Y\right)$ in terms of the vertex function $E$ defined in Eq. (3.5). Thus one can see that the calculation of the triple Pomeron vertex in terms of the functions $g(\underline{k}, \underline{q}, n, \nu)$ is reduced to the calculation of

$$
\frac{2 \pi \bar{\alpha}_{S}^{2}}{N_{c}} \int \frac{d^{2} \underline{x}_{1} d^{2} \underline{x}_{2} d^{2} \underline{x}_{3}}{\underline{x}_{12}^{2} \underline{x}_{23}^{2} \underline{x}_{31}^{2}}\left(L_{12} E\left(\underline{x}_{1}, \underline{x}_{2} ; \underline{x}_{01} \mid n_{1} ; \nu_{1}\right)\right) E^{*}\left(\underline{x}_{2}, \underline{x}_{3} ; \underline{x}_{02} \mid n_{2} ; \nu_{2}\right) E^{*}\left(\underline{x}_{3}, \underline{x}_{1} ; \underline{x}_{03} \mid n_{3} ; \nu_{3}\right)
$$

then being properly convoluted with other necessary vertex functions $E$ as defined in Eq. (3.5) to reproduce Eq. (3.17). We use the fact that the vertex function $E$ are eigenfunctions of the operator $L_{12}$ (see Eq. (3.9)) to recast Eq. (3.18) as

$$
\frac{2 \pi \bar{\alpha}_{S}^{2}}{N_{c}} \int \frac{d^{2} \underline{x}_{1} d^{2} \underline{x}_{2} d^{2} \underline{x}_{3}}{\underline{x}_{12}^{2} \underline{x}_{23}^{2} \underline{x}_{31}^{2}} \frac{1}{\lambda\left(n_{1}, \nu_{1}\right)} E\left(\underline{x}_{1}, \underline{x}_{2} ; \underline{x}_{01} \mid n_{1} ; \nu_{1}\right) E^{*}\left(\underline{x}_{2}, \underline{x}_{3} ; \underline{x}_{02} \mid n_{2} ; \nu_{2}\right) E^{*}\left(\underline{x}_{3}, \underline{x}_{1} ; \underline{x}_{03} \mid n_{3} ; \nu_{3}\right)
$$


Following the standard procedure we used above in finding the Pomeron Green function in terms of functions $g(\underline{k}, \underline{q}, n, \nu)$ we insert the inverse Fourier transform of Eq. (3.14) into Eq. (3.19) and then integrate over the coordinates $\underline{x}_{1}, \underline{x}_{2}$ and $\underline{x}_{3}$ as follows.

$$
\begin{aligned}
& \frac{2 \pi \bar{\alpha}_{S}^{2}}{N_{c}} \int \frac{d^{2} \underline{x}_{1} d^{2} \underline{x}_{2} d^{2} \underline{x}_{3}}{\underline{x}_{12}^{2} \underline{x}_{23}^{2} \underline{x}_{31}^{2}} \frac{1}{\lambda\left(n_{1}, \nu_{1}\right)} \quad \underline{x}_{12}^{2} \int \frac{d^{2} \underline{k}_{12}}{(2 \pi)^{2}} \frac{d^{2} \underline{q}_{1}}{(2 \pi)^{2}} e^{-i \underline{k}_{12} \cdot \underline{x}_{12}} e^{-i \underline{q}_{1} \cdot \underline{z}_{12}} g\left(\underline{k}_{12}, \underline{q}_{1}, n_{1}, \nu_{1}\right) \times \\
& \underline{x}_{23}^{2} \int \frac{d^{2} \underline{k}_{23}}{(2 \pi)^{2}} \frac{d^{2} \underline{q}_{2}}{(2 \pi)^{2}} e^{+i \underline{k}_{23} \cdot \underline{x}_{23}} e^{+i \underline{q}_{2} \cdot \underline{z}_{23}} g^{*}\left(\underline{k}_{23}, \underline{q}_{2}, n_{2}, \nu_{2}\right) \times \\
& \underline{x}_{31}^{2} \int \frac{d^{2} \underline{k}_{31}}{(2 \pi)^{2}} \frac{d^{2} \underline{q}_{3}}{(2 \pi)^{2}} e^{+i \underline{k}_{31} \cdot \underline{x}_{31}} e^{+i \underline{q}_{3} \cdot \underline{z}_{31}} g^{*}\left(\underline{k}_{31}, \underline{q}_{3}, n_{3}, \nu_{3}\right)= \\
& \frac{2 \pi \bar{\alpha}_{S}^{2}}{N_{c}} \int \frac{d^{2} \underline{x}_{1} d^{2} \underline{x}_{2} d^{2} \underline{x}_{3}}{\lambda\left(n_{1}, \nu_{1}\right)} \int \frac{d^{2} \underline{k}_{12}}{(2 \pi)^{2}} \frac{d^{2} \underline{q}_{1}}{(2 \pi)^{2}} e^{-i \underline{k}_{12} \cdot\left(\underline{x}_{1}-\underline{x}_{2}\right)} e^{-i \underline{q}_{1} \cdot \frac{\underline{x}_{1}+\underline{x}_{2}}{2}} e^{+i \underline{q}_{1} \cdot \underline{x}_{01}} g\left(\underline{k}_{12}, \underline{q}_{1}, n_{1}, \nu_{1}\right) \times \\
& \int \frac{d^{2} \underline{k}_{23}}{(2 \pi)^{2}} \frac{d^{2} \underline{q}_{2}}{(2 \pi)^{2}} e^{+i \underline{k}_{23} \cdot\left(\underline{x}_{2}-\underline{x}_{3}\right)} e^{+i \underline{q}_{2} \cdot \frac{\underline{x}_{2}+\underline{x}_{3}}{2}} e^{-i \underline{q}_{2} \cdot \underline{x}_{02}} g^{*}\left(\underline{k}_{23}, \underline{q}_{2}, n_{2}, \nu_{2}\right) \times \\
& \int \frac{d^{2} \underline{k}_{31}}{(2 \pi)^{2}} \frac{d^{2} \underline{q}_{3}}{(2 \pi)^{2}} e^{+i \underline{k}_{31} \cdot\left(\underline{x}_{3}-\underline{x}_{1}\right)} e^{+i \underline{q}_{3} \cdot \frac{\underline{x}_{3}+\underline{x}_{1}}{2}} e^{-i \underline{q}_{3} \cdot \underline{x}_{03}} g^{*}\left(\underline{k}_{31}, \underline{q}_{3}, n_{3}, \nu_{3}\right)
\end{aligned}
$$

The integration over coordinates in Eq. (3.21) gives

$$
\begin{aligned}
& \int d^{2} \underline{x}_{1} \quad \Longrightarrow \quad(2 \pi)^{2} \delta^{(2)}\left(\underline{k}_{12}+\frac{1}{2} \underline{q}_{1}+\underline{k}_{31}-\frac{1}{2} \underline{q}_{3}\right) \\
& \int d^{2} \underline{x}_{2} \quad \Longrightarrow \quad(2 \pi)^{2} \delta^{(2)}\left(-\underline{k}_{12}+\frac{1}{2} \underline{q}_{1}-\underline{k}_{23}-\frac{1}{2} \underline{q}_{2}\right) \\
& \int d^{2} \underline{x}_{3} \quad \Longrightarrow \quad(2 \pi)^{2} \delta^{(2)}\left(\underline{k}_{23}-\frac{1}{2} \underline{q}_{2}-\underline{k}_{31}-\frac{1}{2} \underline{q}_{3}\right)
\end{aligned}
$$

Now we perform the integration over $\underline{k}_{23}, \underline{k}_{31}$ and $\underline{q}_{3}$ which results into

$$
\begin{array}{rlcll}
\int d^{2} \underline{k}_{31} & \Longrightarrow & \underline{k}_{31}=\frac{1}{2} \underline{q}_{3}-\underline{k}_{12}-\frac{1}{2} \underline{q}_{1} \quad \text { and } \quad(2 \pi)^{2} \delta^{(2)}\left(\frac{1}{2} \underline{q}_{3}-\underline{k}_{12}-\frac{1}{2} \underline{q}_{1}+\frac{1}{2} \underline{q}_{2}-\underline{k}_{23}\right) \\
\int d^{2} \underline{k}_{23} & \Longrightarrow & \underline{k}_{23}=\frac{1}{2} \underline{q}_{3}-\underline{k}_{12}-\frac{1}{2} \underline{q}_{1}+\frac{1}{2} \underline{q}_{2} & \text { and } \quad(2 \pi)^{2} \delta^{(2)}\left(\underline{q}_{1}-\underline{q}_{2}-\underline{q}_{3}\right) \\
\int d^{2} \underline{q}_{3} & \Longrightarrow & \underline{q}_{3}=\underline{q}_{1}-\underline{q}_{2} &
\end{array}
$$

where we used the identity $\int d x f(x) \delta(x-a) \delta(x-b)=f(a) \delta(a-b)$. The result of Eq. (3.23) can be summarized as

$$
\begin{aligned}
\underline{k}_{31} & =-\underline{k}_{12}-\frac{1}{2} \underline{q}_{2} \\
\underline{k}_{23} & =-\underline{k}_{12}+\frac{1}{2} \underline{q}_{1}-\frac{1}{2} \underline{q}_{2} \\
\underline{q}_{3} & =\underline{q}_{1}-\underline{q}_{2}
\end{aligned}
$$

Finally, we are in position to write the triple Pomeron vertex in terms of functions $g(\underline{k}, \underline{q}, n, \nu)$. To do this we remove the performed integration over $\underline{x}_{1}, \underline{x}_{2}, \underline{x}_{3}, \underline{k}_{31}, \underline{k}_{23}$ and $\underline{q}_{3}$ in Eq. (3.21) as was shown above by replacing $\underline{k}_{31}, \underline{k}_{23}$ and 
$\underline{q}_{3}$ with the expressions given in Eq. (3.24), as well as, multiply Eq. (3.21) by $(2 \pi)^{6}$ from $\delta$-functions. Thus Eq. (3.21) after the outlined integrations reads

$$
\frac{d^{2} \underline{k}_{12}}{(2 \pi)^{2}} \frac{d^{2} \underline{q}_{1}}{(2 \pi)^{2}} \frac{d^{2} \underline{q}_{2}}{(2 \pi)^{2}} e^{+i \underline{q}_{1} \cdot \underline{x}_{01}-i \underline{q}_{2} \cdot \underline{x}_{02}-i\left(\underline{q}_{1}-\underline{q}_{2}\right) \cdot \underline{x}_{03}} \times \Gamma\left(k_{12}, \underline{q}_{1}, \underline{q}_{2}\right)
$$

where

$$
\Gamma\left(k_{12}, \underline{q}_{1}, \underline{q}_{2}\right)=\frac{2 \pi \bar{\alpha}_{S}^{2}}{N_{c}} \frac{1}{\lambda\left(n_{1}, \nu_{1}\right)} g\left(\underline{k}_{12}, \underline{q}_{1}, n_{1}, \nu_{1}\right) g^{*}\left(-\underline{k}_{12}+\frac{1}{2} \underline{q}_{1}-\frac{1}{2} \underline{q}_{2}, \underline{q}_{2}, n_{2}, \nu_{2}\right) g^{*}\left(-\underline{k}_{12}-\frac{1}{2} \underline{q}_{2}, \underline{q}_{1}-\underline{q}_{2}, n_{3}, \nu_{3}\right)
$$

The expression in Eq. (3.25) represents the triple Pomeron vertex in terms of functions $g(\underline{k}, \underline{q}, n, \nu)$. As it was shown on the example of the free Pomeron propagator, the triple Pomeron vertex should be properly convoluted with all the necessary vertex functions $E$ to give the amplitude of a dipole being scattered off two dipoles. The only missing thing is the explicit expression for $g(\underline{k}, \underline{q}, n, \nu)$ which is calculated in the Appendix A and should be plugged into Eq. (3.16) and Eq. (3.25) for obtaining the final expression. In the region of $4 k^{2} \gg q^{2}$ the function $g(\underline{k}, \underline{q}, 0, \nu)$ is independent of $q^{2}$ and has the form (see Appendix A)

$$
g(\underline{k}, \underline{q}, 0, \nu) \approx C(\nu)\left(k^{2}\right)^{-\frac{1}{2}+i \nu}
$$

where the constant $C(\nu)$ is given by

$$
C(\nu)=\frac{\pi^{2}}{-i \nu} 2^{2 i \nu} \frac{\Gamma^{2}(1 / 2-i \nu) \Gamma^{2}(i \nu)}{\Gamma^{2}(1 / 2+i \nu) \Gamma^{2}(-i \nu)}
$$

\subsection{The simplest 'fan' diagram}

In this section we calculate the set of diagrams shown in Fig. 1, namely, single Pomeron exchange and the first 'fan' diagram for the triple Pomeron interaction.

\subsubsection{The single Pomeron exchange}

According to our definition of the function $g(\underline{k}, q, n, \nu)$ given in Eq. (3.13) the momenta $\underline{k}$ and $\underline{q}$ denote momenta conjugate to the size and the impact parameter of the dipole respectively (see also the remark after Eq. (3.16)). Using this notation we readily write the expression for the dipole with rapidity $Y$ and the transverse momentum $\underline{k}$ being scattered off the dipole with rapidity $Y^{\prime}$ and the transverse momentum $\underline{k}_{0}$ due to the exchange of one Pomeron.

$$
\begin{aligned}
A(1 P) & =\int d \nu g^{*}(\underline{k}, \underline{q}, 0, \nu) \nu^{2} \lambda(0, \nu) g\left(\underline{k}_{0}, \underline{q}, 0, \nu\right) e^{\omega(\nu)\left(Y-Y^{\prime}\right)} \\
& =4^{2} \pi^{4} \int d \nu \frac{1}{k k_{0}}\left(\frac{k_{0}}{k}\right)^{2 i \nu} e^{\omega_{0}(\nu)\left(Y-Y^{\prime}\right)}=32 \pi^{6} P\left(k_{0} ; k \mid Y-Y^{\prime}\right)
\end{aligned}
$$

where $\omega_{0}(\nu) \approx 4 \ln 2-14 \zeta(2) \nu^{2}$ is the expansion of the BFKL eigenvalue in the vicinity of $\nu=0$ bringing the main contribution at high energies, and $P\left(k_{0} ; k \mid Y-Y^{\prime}\right)$ is well known expression for single Pomeron exchange given by

$$
P\left(k_{0} ; k \mid Y-Y^{\prime}\right)=\frac{1}{\pi} \int \frac{d \nu}{2 \pi} \frac{1}{k k_{0}}\left(\frac{k_{0}}{k}\right)^{2 i \nu} e^{\omega_{0}(\nu)\left(Y-Y^{\prime}\right)}
$$

The factor of $32 \pi^{6}$ merely reflects our normalization of functions $g(\underline{k}, \underline{q}, 0, \nu)$ defined in Eq. (3.13). From Eq. (3.29) one can see that the anomalous dimension of the gluon structure function $\left(x G\left(x, Q^{2}\right) \propto\left(Q^{2}\right)^{\gamma}\right)$ is equal to $\gamma=\frac{1}{2}-i \nu$ since $P\left(k_{0} ; k \mid Y-Y^{\prime}\right) \propto\left(1 / k^{2}\right) x G\left(x=\exp \left(Y-Y^{\prime}\right), k^{2} / k_{0}^{2}\right)$. 


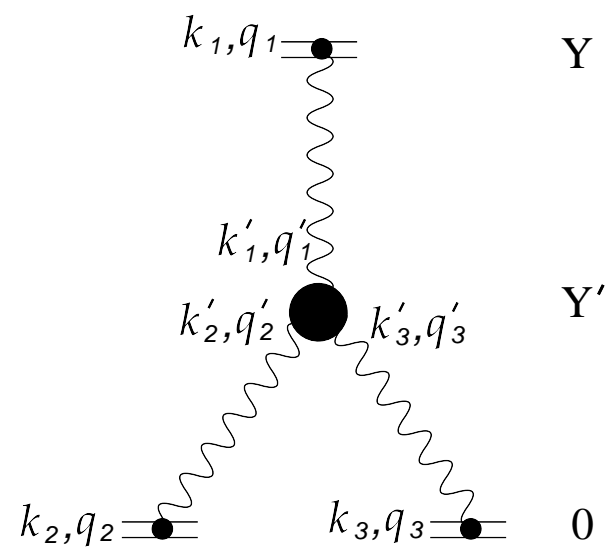

Figure 4: The first 'fan' diagram. Wave lines denote the BFKL Pomerons.

\subsubsection{The first 'fan' diagram}

The first fan diagram, for interaction of one dipole with two dipoles, is shown in Fig. A. Using the representation for triple Pomeron vertex in momentum space calculated in Eq. 3.25$)$ we can write the amplitude corresponding to Fig. 嘈 for $n=0$ as

$$
\begin{aligned}
-A(P \rightarrow 2 P ; \text { Fig. 国 })= & \frac{2 \pi \bar{\alpha}_{s}^{2}}{N_{c}} \int_{0}^{Y} d Y^{\prime} \int \frac{d^{2} \underline{k}_{1}^{\prime}}{(2 \pi)^{2}} \frac{d^{2} \underline{q}_{1}^{\prime}}{(2 \pi)^{2}} \frac{d^{2} \underline{q}_{2}^{\prime}}{(2 \pi)^{2}} d \nu_{1} d \nu_{2} d \nu_{3}(2 \pi)^{2} \delta^{(2)}\left(\underline{q}_{1}-\underline{q}_{1}^{\prime}\right)(2 \pi)^{2} \delta^{(2)}\left(\underline{q}_{2}-\underline{q}_{2}^{\prime}\right) \times \\
& (2 \pi)^{2} \delta^{(2)}\left(\underline{q}_{3}-\underline{q}_{1}^{\prime}+\underline{q}_{2}^{\prime}\right) \frac{\nu_{1}^{2} \nu_{2}^{2} \nu_{3}^{3} \lambda\left(0, \nu_{1}\right) \lambda\left(0, \nu_{2}\right) \lambda\left(0, \nu_{3}\right)}{\lambda\left(0, \nu_{1}\right)} e^{\omega\left(\nu_{1}\right)\left(Y-Y^{\prime}\right)} e^{\omega\left(\nu_{2}\right)\left(Y^{\prime}-0\right)} \times(3.30) \\
& e^{\omega\left(\nu_{3}\right)\left(Y^{\prime}-0\right)} g^{*}\left(\underline{k}_{1}, \underline{q}_{1}, 0, \nu_{1}\right) g\left(\underline{k}_{1}^{\prime}, \underline{q}_{1}^{\prime}, 0, \nu_{1}\right) g^{*}\left(-\underline{k}_{1}^{\prime}+\frac{1}{2} \underline{q}_{1}^{\prime}-\frac{1}{2} \underline{q}_{2}^{\prime}, \underline{q}_{1}^{\prime}, 0, \nu_{2}\right) g\left(\underline{k}_{2}, \underline{q}_{2}, 0, \nu_{2}\right) \times \\
& g^{*}\left(-\underline{k}_{1}^{\prime}-\frac{1}{2} \underline{q}_{2}^{\prime}, \underline{q}_{1}^{\prime}-\underline{q}_{2}^{\prime}, 0, \nu_{3}\right) g\left(\underline{k}_{3}, \underline{q}_{3}, 0, \nu_{3}\right)
\end{aligned}
$$

where $\underline{k}_{2}^{\prime}=-\underline{k}_{1}^{\prime}+\frac{1}{2} \underline{q}_{1}^{\prime}-\frac{1}{2} \underline{q}_{2}^{\prime}, \underline{k}_{3}^{\prime}=-\underline{k}_{1}^{\prime}-\frac{1}{2} \underline{q}_{2}^{\prime}$ and $\underline{q}_{3}^{\prime}=\underline{q}_{1}^{\prime}-\underline{q}_{2}^{\prime}$ as it was shown in the derivation of Eq. (3.25).

For the case of small momenta transferred along the Pomeron we approximate the vertex function by expression given in Eq. (3.26). It is worth to mention that the constants $C(\nu)$ defined in Eq. (3.27) have a property

$$
C(\nu) C^{*}(\nu)=\frac{\pi^{4}}{\nu^{2}} \quad \text { for } \quad \nu \in \mathbb{R}
$$

Thus Eq. (3.30) reads

$$
\begin{aligned}
-A(P \rightarrow 2 P ; \text { Fig. 目 })= & \frac{2 \pi \bar{\alpha}_{s}^{2}}{N_{c}} \int_{0}^{Y} d Y^{\prime} \int \frac{d^{2} \underline{k}_{1}^{\prime}}{(2 \pi)^{2}} \frac{d^{2} \underline{q}_{1}^{\prime}}{(2 \pi)^{2}} \frac{d^{2} \underline{q}_{2}^{\prime}}{(2 \pi)^{2}} d \nu_{1} d \nu_{2} d \nu_{3}(2 \pi)^{2} \delta^{(2)}\left(\underline{q}_{1}-\underline{q}_{1}^{\prime}\right)(2 \pi)^{2} \delta^{(2)}\left(\underline{q}_{2}-\underline{q}_{2}^{\prime}\right) \times(3.32) \\
& (2 \pi)^{2} \delta^{(2)}\left(\underline{q}_{3}-\underline{q}_{1}^{\prime}+\underline{q}_{2}^{\prime}\right)\left(\pi^{4}\right)^{3} \lambda\left(0, \nu_{2}\right) \lambda\left(0, \nu_{3}\right) e^{\omega\left(\nu_{1}\right)\left(Y-Y^{\prime}\right)} e^{\omega\left(\nu_{2}\right)\left(Y^{\prime}-0\right)} e^{\omega\left(\nu_{3}\right)\left(Y^{\prime}-0\right)} \times \\
& \left|\underline{k}_{1}\right|^{-1-2 i \nu_{1}}\left|\underline{k}_{1}^{\prime}\right|^{-1+2 i \nu_{1}}\left|-\underline{k}_{1}^{\prime}+\underline{q}_{1}^{\prime} / 2-\underline{q}_{2}^{\prime} / 2\right|^{-1-2 i \nu_{2}}\left|\underline{k}_{2}\right|^{-1+2 i \nu_{2}}\left|-\underline{k}_{1}^{\prime}-\underline{q}_{2}^{\prime} / 2\right|^{-1-2 i \nu_{3}}\left|\underline{k}_{3}\right|^{-1+2 i \nu_{3}}
\end{aligned}
$$

In Eq. (3.32) momenta $\underline{q}_{1}$ and $\underline{q}_{2}$ are arbitrary. However, in the mean field approximation (MFA) the momenta 
transferred along the Pomeron are much smaller than momenta conjugate to dipole sizes ${ }^{2}|\underline{k}| \gg|\underline{q}|$, which allows us to simply Eq. (3.32) using $\left|-\underline{k}_{1}^{\prime}+\underline{q}_{1}^{\prime} / 2-\underline{q}_{2}^{\prime} / 2\right| \approx\left|\underline{k}_{1}^{\prime}\right|$ and $\left|-\underline{k}_{1}^{\prime}-\underline{q}_{2}^{\prime} / 2\right| \approx\left|\underline{k}_{1}^{\prime}\right|$. It is easy to see that the integration over $\underline{k}_{1}^{\prime}$ leads to a delta function $\delta\left(i+2 \nu_{1}-2 \nu_{2}-2 \nu_{3}\right)$, which is the conservation of the anomalous dimensions $(\gamma=1 / 2-i \nu)$ in the triple Pomeron vertex $\delta\left(1+\gamma_{1}-\gamma_{2}-\gamma_{3}\right)$ (see Ref. 15 where this $\delta$-function was found for the forward scattering amplitude). However we need to be careful since in Eq. (3.32) we implicitly used the condition that $k_{3}^{2}<k_{1}^{\prime 2}<k_{1}^{2}$. For $k_{1}^{\prime 2}>k_{1}^{2}$ the second term in Eq. (A-21) gives the main contribution. Therefore we integrate over all the kinematic region from ${k^{\prime}}_{1}^{2}=0$ to ${k^{\prime}}_{1}^{2}=\infty$ but subtract the regions ${k^{\prime}}_{1}^{2}>k_{1}^{2}$ and replace them by correct integral.

For example for ${k_{1}^{\prime}}_{1}^{2}>k_{1}^{2}$ we obtain the following integral

$$
\left(\frac{k_{1}^{2}}{k_{3}^{2}}\right)^{-i \nu_{1}} \int_{l}^{\infty} d l^{\prime} e^{\left(-1+i 2 \nu_{1}-2 i \nu_{2}-2 i \nu_{3}\right) l^{\prime}}-\left(\frac{k_{1}^{2}}{k_{3}^{2}}\right)^{i \nu_{1}} \int_{l}^{\infty} d l^{\prime} e^{\left(-1-i 2 \nu_{1}-2 i \nu_{2}-2 i \nu_{3}\right) l^{\prime}}
$$

where $l=\ln \left(k_{1}^{2} / k_{3}^{2}\right)$. The result of the integration leads to

$$
e^{\left(-1-i \nu_{2}-i \nu_{3}\right) l}\left(\frac{1}{-1+i 2 \nu_{1}-i 2 \nu_{2}-2 i \nu_{3}}-\frac{1}{-1-i 2 \nu_{1}-i 2 \nu_{2}-2 i \nu_{3}}\right)
$$

It is easy to see that the integral over $\nu_{1}$ is equal to zero. The behaviour of $\omega\left(\nu_{1}\right)$ at large values of $\nu_{1}$ we regularise by adding a small $i \epsilon$ term for $\nu_{1}\left(\omega\left(\nu_{1}\right) \rightarrow \omega\left(\nu_{1}+i \epsilon\right) \cdot \omega\left(\nu_{1}+i \epsilon\right) \stackrel{\nu_{1} \rightarrow i \epsilon \pm \infty}{\longrightarrow}-\infty\right.$ providing a good convergence of the integral on the large circle.

Therefore, finally we reproduce the $\delta\left(\gamma_{1}-\gamma_{2}-\gamma_{3}\right)$ contribution.

After further integration over $\underline{q}_{1}, \underline{q}_{2}$ and $d \nu_{1}$ in Eq. 3.32 we obtain

$$
\begin{aligned}
-A(P \rightarrow 2 P ; F i g \text {. 国 })= & \frac{2 \pi \bar{\alpha}_{s}^{2}}{N_{c}} \int_{0}^{Y} d Y^{\prime} \int d \nu_{2} d \nu_{3} \frac{1}{2}(2 \pi)^{2} \delta^{(2)}\left(\underline{q}_{3}-\underline{q}_{1}+\underline{q}_{2}\right)\left(\pi^{4}\right)^{3} \lambda\left(0, \nu_{2}\right) \lambda\left(0, \nu_{3}\right) \times \\
& e^{\omega\left(i / 2-\nu_{2}-\nu_{3}\right)\left(Y-Y^{\prime}\right)} e^{\omega\left(\nu_{2}\right)\left(Y^{\prime}-0\right)} e^{\omega\left(\nu_{3}\right)\left(Y^{\prime}-0\right)}\left|\underline{k}_{1}\right|^{-1-2 i \nu_{2}-1-2 i \nu_{3}}\left|\underline{k}_{2}\right|^{-1+2 i \nu_{2}}\left|\underline{k}_{3}\right|^{-1+2 i \nu_{3}} \\
= & \frac{2 \pi \bar{\alpha}_{s}^{2}}{N_{c}} \int d \nu_{2} d \nu_{3} \frac{1}{2}(2 \pi)^{2} \delta^{(2)}\left(\underline{q}_{3}-\underline{q}_{1}+\underline{q}_{2}\right) \frac{\left(\pi^{4}\right)^{3} \lambda\left(0, \nu_{2}\right) \lambda\left(0, \nu_{3}\right)}{\omega\left(-i / 2+\nu_{2}+\nu_{3}\right)-\omega\left(\nu_{2}\right)-\omega\left(\nu_{3}\right)} \times(3.35) \\
& \left|\underline{k}_{1}\right|^{-1-2 i \nu_{2}-1-2 i \nu_{3}}\left|\underline{k}_{2}\right|^{-1+2 i \nu_{2}}\left|\underline{k}_{3}\right|^{-1+2 i \nu_{3}}\left(e^{\omega\left(-i / 2+\nu_{2}+\nu_{3}\right)(Y-0)}-e^{\left(\omega\left(\nu_{2}\right)+\omega\left(\nu_{3}\right)\right)(Y-0)}\right)
\end{aligned}
$$

As was already shown in the toy model in Eq. 2.1) the integration over rapidity brings in two terms in the last line of Eq. (3.35). The first term corresponds to single Pomeron exchange and the second term corresponds to double Pomeron exchange of two non-interacting Pomerons. To see this we rewrite the two terms in a more convenient form. The first term in the last line of Eq. (3.35) reads

$$
\begin{aligned}
\frac{2 \pi \bar{\alpha}_{s}^{2}}{N_{c}} \frac{1}{2}(2 \pi)^{2} \delta^{(2)}\left(\underline{q}_{3}-\underline{q}_{1}+\underline{q}_{2}\right) & \int d \nu_{2} d \mu \frac{\left(\pi^{4}\right)^{3} \lambda\left(0, \nu_{2}\right) \lambda\left(0, i / 2+\mu-\nu_{2}\right)}{\omega(\mu)-\omega\left(\nu_{2}\right)-\omega\left(i / 2+\mu-\nu_{2}\right)} \times \\
& \left|\underline{k}_{1}\right|^{-1-2 i \mu}\left|\underline{k}_{2}\right|^{-1+2 i \nu_{2}}\left|\underline{k}_{3}\right|^{-1+2 i \mu-1-2 i \nu_{2}} e^{\omega(\mu)(Y-0)}
\end{aligned}
$$

\footnotetext{
${ }^{2}$ To see this It is enough to recall that we can safely use MFA for the nucleus target where $k_{1} \approx k_{2} \approx 1 / R$ but $q_{1} \approx q_{2} \approx 1 / R_{A}$ where $R$ and $R_{A}$ are nucleon and nucleus radii and $R_{A} \gg R$.
} 
where $\mu=-i / 2+\nu_{2}+\nu_{3}$. At high energies the leading contribution comes from small values of $\mu$ and we can simplify Eq. (3.36) as

$$
\begin{gathered}
\frac{2 \pi \bar{\alpha}_{s}^{2}}{N_{c}} \frac{1}{2}(2 \pi)^{2} \delta^{(2)}\left(\underline{q}_{3}-\underline{q}_{1}+\underline{q}_{2}\right) \int d \nu_{2} \frac{\left(\pi^{4}\right)^{3} \lambda\left(0, \nu_{2}\right) \lambda\left(0,-\nu_{2}+i / 2\right)}{\omega(0)-\omega\left(\nu_{2}\right)-\omega\left(-\nu_{2}+i / 2\right)} \frac{1}{k_{2} k_{3}}\left(\frac{k_{3}}{k_{2}}\right)^{2 i \nu_{2}} \int d \mu \frac{1}{k_{1} k_{3}}\left(\frac{k_{3}}{k_{1}}\right)^{2 i \mu} e^{\omega(\mu)(Y-0)}= \\
\frac{2 \pi \bar{\alpha}_{s}^{2}}{N_{c}} \frac{1}{2}(2 \pi)^{2} \delta^{(2)}\left(\underline{q}_{3}-\underline{q}_{1}+\underline{q}_{2}\right) f\left(k_{2}, k_{3}\right) 2 \pi^{2} P\left(k_{3} ; k_{1} \mid Y-0\right)
\end{gathered}
$$

where $P\left(k_{3} ; k_{1} \mid Y-0\right)$ is a single Pomeron exchange defined in Eq. (3.29) and $f\left(k_{2}, k_{3}\right)$ is a function of $k_{2}$ and $k_{3}$ which has to be calculated and is given by

$$
f\left(k_{2}, k_{3}\right)=\int d \nu_{2} \frac{\left(\pi^{4}\right)^{3} \lambda\left(0, \nu_{2}\right) \lambda\left(0,-\nu_{2}+i / 2\right)}{\omega(0)-\omega\left(\nu_{2}\right)-\omega\left(-\nu_{2}+i / 2\right)} \frac{1}{k_{2} k_{3}}\left(\frac{k_{3}}{k_{2}}\right)^{2 i \nu_{2}}
$$

The second term of Eq. (3.35) represents two non-interacting Pomeron exchange. To see this we recast it in the form of

$$
\begin{aligned}
& \frac{2 \pi \bar{\alpha}_{s}^{2}}{N_{c}} \int d \nu_{2} d \nu_{3} \frac{1}{2}(2 \pi)^{2} \delta^{(2)}\left(\underline{q}_{3}-\underline{q}_{1}+\underline{q}_{2}\right) \frac{\left(\pi^{4}\right)^{3} \lambda\left(0, \nu_{2}\right) \lambda\left(0, \nu_{3}\right)}{\omega\left(-i / 2+\nu_{2}+\nu_{3}\right)-\omega\left(\nu_{2}\right)-\omega\left(\nu_{3}\right)} \times \\
& \frac{1}{k_{1} k_{2}}\left(\frac{k_{2}}{k_{1}}\right)^{2 i \nu_{2}} e^{\omega\left(\nu_{2}\right)(Y-0)} \frac{1}{k_{1} k_{3}}\left(\frac{k_{3}}{k_{1}}\right)^{2 i \nu_{3}} e^{\omega\left(\nu_{3}\right)(Y-0)}
\end{aligned}
$$

At high energies the main contribution comes from small values of $\nu_{2}$ and $\nu_{3}$ and thus we can simplify Eq. (3.39) as follows

$$
\frac{2 \pi \bar{\alpha}_{s}^{2}}{N_{c}} \frac{1}{2}(2 \pi)^{2} \delta^{(2)}\left(\underline{q}_{3}-\underline{q}_{1}+\underline{q}_{2}\right)\left(4^{2}\right)^{2} \frac{\left(\pi^{4}\right)^{3}}{\omega(-i / 2)-\omega(0)-\omega(0)}\left(2 \pi^{2}\right)^{2} P\left(k_{2}, k_{1} \mid Y-0\right) P\left(k_{3}, k_{1} \mid Y-0\right)
$$

Comparing Eq. (3.35) with Eq. (2.1) using the last result we conclude that in QCD the 'fan' diagrams can be viewed as the contribution from the exchange of two non-interacting BFKL Pomerons and a renormalisation of the single Pomeron contribution.

However, as it was noticed by Hatta and Mueller [44], there is another saddle point in Eq. (3.35) where the denominator $\omega\left(\nu_{2}+\nu_{3}-i / 2\right)-\omega\left(\nu_{2}\right)-\omega\left(\nu_{3}\right)$ is close to zero. Indeed, the equation for this saddle point $\left(\gamma_{S P}=\right.$ $\left.1 / 2-i \nu_{S P, 1}\right)$ looks as follows

$$
2 \omega^{\prime}\left(\gamma_{S P}\right) Y-\frac{2\left(\omega ^ { \prime } \left(2\left(\gamma_{S P}-1\right)-\omega^{\prime}\left(\gamma_{S P}\right)\right.\right.}{\omega\left(2 \gamma_{S P}-1\right)-2 \omega\left(\gamma_{S P}\right)}+\ln \left(k_{1}^{2} / k_{3}^{2}\right)=0
$$

In Eq. (3.42) we assume that the main contribution is dominated by $\nu_{1}=\nu_{2}$ One can see that the position of this saddle point is very close to the solution of the equation

$$
\omega\left(2 \gamma_{0}-1\right)-2 \omega\left(\gamma_{0}\right)=0
$$

Denoting $\gamma_{S P}-\gamma_{0}$ by $\delta \gamma$ one can see from Eq. (3.42) that for the first term in Eq. (3.35) we have

$$
\delta \gamma=\frac{1}{2 \omega\left(\gamma_{0}\right) Y+\ln \left(k_{1}^{2} / k_{3}^{2}\right)} \ll \gamma_{0}
$$

and for the second one

$$
\delta \gamma=\frac{1}{2 \omega\left(2 \gamma_{0}-1\right) Y+\ln \left(k_{1}^{2} / k_{3}^{2}\right)} \ll \gamma_{0}
$$




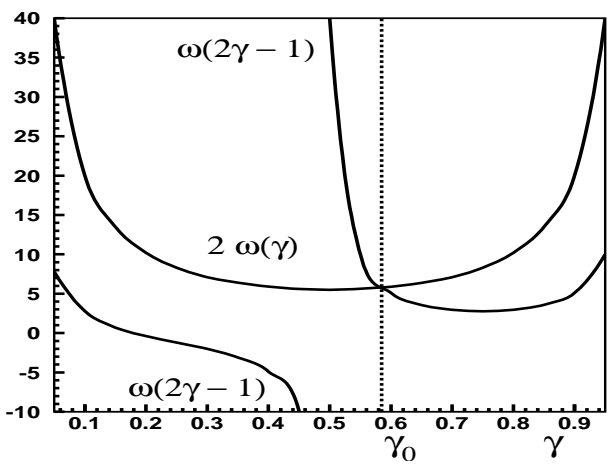

Figure 5: Solution to Eq. 3.43 for $\omega$ 's, given by the BFKL equations.

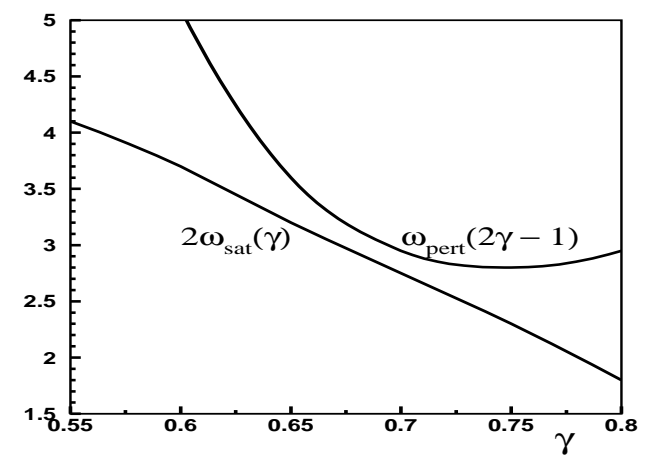

Figure 6: Solution to Eq. 3.43) for $\omega_{\text {pert }}$ given by the BFKL equation and $\omega_{\text {sat }}$ calculated from Eq. (3.47).

The sum of these two terms leads to the contribution with energy dependence

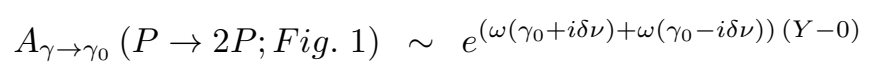

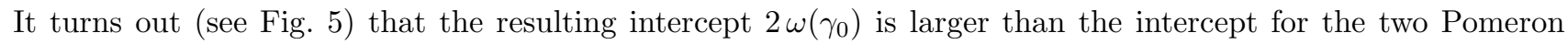
exchange $(2 \omega(\gamma=1 / 2))$ and, therefore, this contribution is the largest among the three. The appearance of such new singularities in the angular momentum plane is a deadly blow to the entire approach based on the BFKL Pomeron. Indeed, since the new singularity (double pole) is located to the right of the singularity generated by the exchange of two BFKL Pomerons, we need firstly to sum over all such singularities, to obtain the resulting Green function and only after doing this can we build the Reggeon calculus based on this Green function. However, the situation changes crucially if we take into account the fact that in Eq. (3.43) $\omega\left(\gamma_{0}\right)$ and $\omega\left(2 \gamma_{0}-1\right)$ are actually in quite different kinematic regions. $\omega(2 \gamma-1)$ enters with a small value of the argument, namely $2 \gamma_{0}-1<\gamma_{c r}$ and, therefore, can be calculated using the BFKL equation of Eq. (3.7). We recall that $\gamma_{c r}$ can be found from the following equation

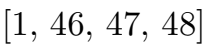

$$
\frac{\chi\left(\gamma_{c r}\right)}{1-\gamma_{c r}}=-\frac{d \chi\left(\gamma_{c r}\right)}{d \gamma_{c r}}
$$

However, we have a different situation for $\omega\left(\gamma_{0}\right)$. Indeed, $\gamma_{0}$ turns out to be larger than $\gamma_{c r}$ (see Fig. 5 and recall that $\left.\gamma_{c r}=0.37\right)$. Therefore, $\omega\left(\gamma_{0}\right)$ describes the behaviour of the scattering amplitude in the saturation region, and, therefore, cannot be calculated using Eq. (3.7) (see also Ref. [45], where the same conclusion has been derived from slightly different considerations). As it was found in Ref. [46] for $\omega$ in the saturation region we have

$$
\omega_{s a t}(\gamma)=\frac{\omega\left(\gamma_{c r}\right)}{1-\gamma_{c r}}(1-\gamma)
$$

at least for $\gamma>\gamma_{c r}$ but close to $\gamma_{c r}$.

As one can see from Fig. 6 Eq. (3.43) which can be written as

$$
2 \omega_{\text {sat }}\left(\gamma_{0}\right)=\omega_{\text {pert }}\left(2 \gamma_{0}-1\right)
$$

has no solution. 


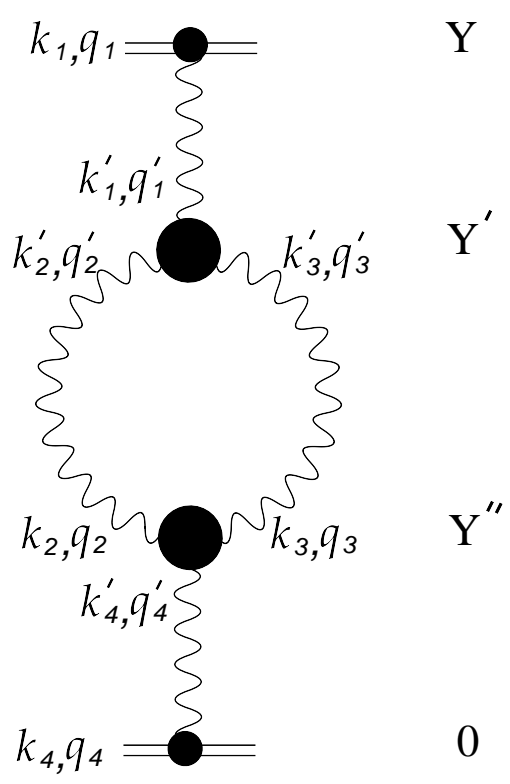

Figure 7: The first enhanced diagram.
Having this observation in mind our suggestion is to ignore this singularity. Our proposed strategy is the following: we ignore all such singularities that appear to be due to the possibility that two or more poles have the same position. Next we solve the problem and return to considering contributions to the scattering amplitude having the solution both in the perturbative QCD region as well as in the saturation region.

Finally, we consider only two contributions for the simplest 'fan' diagram: the first one, given by Eq. (3.35), is due to the exchange of two non-interacting Pomerons; and the second term of 3.35 which is the renormalisation of the amplitude of the Pomeron interaction with the target. Both these terms are the same as what we considered in the BFKL Pomeron calculus in zero transverse dimensions.

\subsection{The first enhanced diagram}

In this subsection we will calculate the simplest enhanced diagram shown in Fig. 7, whose contribution has the form

$$
\begin{aligned}
& -A(P \rightarrow 2 P \rightarrow P \text { F Fig. 团 })=\left(\frac{2 \pi \bar{\alpha}_{s}^{2}}{N_{c}}\right)^{2} \int_{0}^{Y} d Y^{\prime} \int_{0}^{Y^{\prime}} d Y^{\prime \prime} \int \frac{d^{2} \underline{k}_{1}^{\prime}}{(2 \pi)^{2}} \frac{d^{2} \underline{q}_{1}^{\prime}}{(2 \pi)^{2}} \frac{d^{2} \underline{q}_{2}^{\prime}}{(2 \pi)^{2}} \frac{d^{2} \underline{k}_{4}^{\prime}}{(2 \pi)^{2}} \frac{d^{2} \underline{q}_{4}^{\prime}}{(2 \pi)^{2}} \frac{d^{2} \underline{q}_{3}}{(2 \pi)^{2}} \times \\
& (2 \pi)^{2} \delta^{(2)}\left(\underline{q}_{1}-\underline{q}_{1}^{\prime}\right)(2 \pi)^{2} \delta^{(2)}\left(\underline{q}_{3}-\underline{q}_{4}^{\prime}-\underline{q}_{2}^{\prime}\right)(2 \pi)^{2} \delta^{(2)}\left(\underline{q}_{3}-\underline{q}_{1}^{\prime}-\underline{q}_{2}^{\prime}\right)(2 \pi)^{2} \delta^{(2)}\left(\underline{q}_{4}-\underline{q}_{4}^{\prime}\right) \times \\
& \int d \nu_{1} d \nu_{2} d \nu_{3} d \nu_{4} \nu_{1}^{2} \nu_{2}^{2} \nu_{3}^{2} \nu_{4}^{2} \frac{\lambda\left(0, \nu_{1}\right) \lambda\left(0, \nu_{2}\right) \lambda\left(0, \nu_{3}\right) \lambda\left(0, \nu_{4}\right)}{\lambda\left(0, \nu_{1}\right) \lambda\left(0, \nu_{4}\right)} e^{\omega\left(\nu_{1}\right)\left(Y-Y^{\prime}\right)} \times \\
& e^{\omega\left(\nu_{2}\right)\left(Y^{\prime}-Y^{\prime \prime}\right)} e^{\omega\left(\nu_{3}\right)\left(Y^{\prime}-Y^{\prime \prime}\right)} e^{\omega\left(\nu_{4}\right)\left(Y^{\prime \prime}-0\right)} g^{*}\left(\underline{k}_{1}, \underline{q}_{1}, 0, \nu_{1}\right) g\left(\underline{k}_{1}^{\prime}, \underline{q}_{1}^{\prime}, 0, \nu_{1}\right) \times \\
& g^{*}\left(-\underline{k}_{1}^{\prime}+\underline{q}_{1}^{\prime} / 2-\underline{q}_{2}^{\prime} / 2, \underline{q}_{2}^{\prime}, 0, \nu_{2}\right) g\left(-\underline{k}_{4}^{\prime}-\underline{q}_{3} / 2, \underline{q}_{3}-\underline{q}_{4}^{\prime}, 0, \nu_{2}\right) \times \\
& g^{*}\left(-\underline{k}_{1}^{\prime}-\underline{q}_{2}^{\prime} / 2, \underline{q}_{1}^{\prime}-\underline{q}_{2}^{\prime}, 0, \nu_{3}\right) g\left(-\underline{k}_{4}^{\prime}+\underline{q}_{4}^{\prime} / 2-\underline{q}_{3} / 2, \underline{q}_{3}, 0, \nu_{3}\right) \times \\
& g^{*}\left(\underline{k}_{4}^{\prime}, \underline{q}_{4}^{\prime}, 0, \nu_{4}\right) g\left(\underline{k}_{4}, \underline{q}_{4}, 0, \nu_{4}\right) \\
& =\left(\frac{2 \pi \bar{\alpha}_{s}^{2}}{N_{c}}\right)^{2}(2 \pi)^{2} \delta^{(2)}\left(\underline{q}_{1}-\underline{q}_{4}\right) \int_{0}^{Y} d Y^{\prime} \int_{0}^{Y^{\prime}} d Y^{\prime \prime} \int \frac{d^{2} \underline{k}_{1}^{\prime}}{(2 \pi)^{2}} \frac{d^{2} \underline{q}_{2}^{\prime}}{(2 \pi)^{2}} \frac{d^{2} \underline{k}_{4}^{\prime}}{(2 \pi)^{2}} \times \\
& \int d \nu_{1} d \nu_{2} d \nu_{3} d \nu_{4} \nu_{1}^{2} \nu_{2}^{2} \nu_{3}^{2} \nu_{4}^{2} \lambda\left(0, \nu_{2}\right) \lambda\left(0, \nu_{3}\right) e^{\omega\left(\nu_{1}\right)\left(Y-Y^{\prime}\right)} \times \\
& e^{\omega\left(\nu_{2}\right)\left(Y^{\prime}-Y^{\prime \prime}\right)} e^{\omega\left(\nu_{3}\right)\left(Y^{\prime}-Y^{\prime \prime}\right)} e^{\omega\left(\nu_{4}\right)\left(Y^{\prime \prime}-0\right)} g^{*}\left(\underline{k}_{1}, \underline{q}_{1}, 0, \nu_{1}\right) g\left(\underline{k}_{1}^{\prime}, \underline{q}_{1}, 0, \nu_{1}\right) \times \\
& g^{*}\left(-\underline{k}_{1}^{\prime}+\underline{q}_{1} / 2-\underline{q}_{2}^{\prime} / 2, \underline{q}_{2}^{\prime}, 0, \nu_{2}\right) g\left(-\underline{k}_{4}^{\prime}-\underline{q}_{1} / 2-\underline{q}_{2}^{\prime} / 2, \underline{q}_{1} / 2+\underline{q}_{2}^{\prime} / 2-\underline{q}_{4}, 0, \nu_{2}\right) \times \\
& g^{*}\left(-\underline{k}_{1}^{\prime}-\underline{q}_{2}^{\prime} / 2, \underline{q}_{1}-\underline{q}_{2}^{\prime}, 0, \nu_{3}\right) g\left(-\underline{k}_{4}^{\prime}+\underline{q}_{4} / 2-\underline{q}_{1} / 2-\underline{q}_{2}^{\prime} / 2, \underline{q}_{1} / 2-\underline{\nu}_{1}\right) \times \\
& g^{*}\left(\underline{k}_{4}^{\prime}, \underline{q}_{4}, 0, \nu_{4}\right) g\left(\underline{k}_{4}, \underline{q}_{4}, 0, \nu_{4}\right)
\end{aligned}
$$

where we used the fact that $\underline{k}_{3}=-\underline{k}_{4}^{\prime}+\underline{q}_{4}^{\prime} / 2-\underline{q}_{3} / 2, \underline{k}_{2}=-\underline{k}_{4}^{\prime}-\underline{q}_{3} / 2$ and $\underline{q}_{2}=\underline{q}_{3}-\underline{q}_{4}^{\prime}$.

Considering, for simplicity, the case of the forward scattering $\left(\underline{q}_{1}=\underline{q}_{1}=\underline{q}_{4}^{\prime}=\underline{q}_{4}=0\right.$ and denoting $\underline{q}_{2}=\underline{q}_{2}^{\prime}=\underline{q}^{\prime}$ as well as using the following notations: $\nu_{1} \equiv \nu, \nu_{4} \equiv \nu^{\prime}$ and $\nu_{2} \equiv \nu_{1}, \nu_{3} \equiv \nu_{2}$, in $\omega$ representation this diagram 
reduces to the following contribution

$$
\begin{aligned}
-A(\text { Fig. 团 }) & =\left(\frac{2 \pi \bar{\alpha}_{S}^{2}}{N_{c}}\right)^{2} \int \nu^{2} d \nu \prod_{i=1}^{2} \nu_{i}^{2} \lambda\left(\nu_{i}\right) \nu^{\prime 2} d \nu_{i} d \nu^{\prime} d^{2} q^{\prime} d^{2} k_{1} d^{2} k_{2} \\
& \times g^{*}(\underline{k}, 0,0, \nu) g\left(\underline{k}_{1}, 0,0, \nu\right) g^{*}\left(-\underline{k}_{1}^{\prime}+\underline{q}^{\prime} / 2, \underline{q}^{\prime}, 0, \nu_{1}\right) g^{*}\left(-\underline{k}_{1}^{\prime}-\underline{q}^{\prime} / 2,-\underline{q}^{\prime}, 0, \nu_{2}\right) \\
& \times g\left(-\underline{k}_{2}+\underline{q}^{\prime} / 2, \underline{q}^{\prime}, 0, \nu_{1}\right) g^{*}\left(-\underline{k}_{2}-\underline{q}^{\prime} / 2,-\underline{q}^{\prime}, 0, \nu_{2}\right) g^{*}\left(\underline{k}_{4}, 0,0, \nu^{\prime}\right) g\left(\underline{k}_{4}, 0,0, \nu^{\prime}\right) \\
& \times \frac{1}{2 \pi i} \int_{a-i \infty}^{a+i \infty} d \omega e^{\omega Y} \frac{1}{\omega-\omega(\nu)} \frac{1}{\omega-\omega\left(\nu_{1}\right)-\omega\left(\nu_{2}\right)} \frac{1}{\omega-\omega\left(\nu^{\prime}\right)}
\end{aligned}
$$

The integral over $\omega$ can be rewritten as the sum over different contributions. We will show below that $\nu=\nu^{\prime}$ and the integral has the form

$$
\begin{aligned}
& \frac{1}{2 \pi i} \int_{a-i \infty}^{a+i \infty} d \omega e^{\omega Y} \frac{1}{(\omega-\omega(\nu))^{2}} \frac{1}{\omega-\omega\left(\nu_{1}\right)-\omega\left(\nu_{2}\right)} \rightarrow \\
& e^{\omega(\nu) Y}\left(Y-\frac{1}{\omega(\nu)-\omega\left(\nu_{1}\right)-\omega\left(\nu_{2}\right)}\right) \frac{1}{\omega(\nu)-\omega\left(\nu_{1}\right)-\omega\left(\nu_{2}\right)}+e^{\left(\omega\left(\nu_{1}\right)+\omega\left(\nu_{2}\right)\right) Y} \frac{1}{\left(\omega(\nu)-\omega\left(\nu_{1}\right)-\omega\left(\nu_{2}\right)\right)^{2}}
\end{aligned}
$$

The first term leads to the renormalisation of the BFKL Pomeron intercept (the first term in the brackets) as well as of the vertex of the interaction of the BFKL Pomeron with the target (see Fig. (3). The second term reduces to the exchange of two BFKL Pomerons without any interaction between them. However, for $\gamma_{0}=\frac{1}{2}-i \nu_{0}$, given by Eq. (3.43), instead of Eq. (3.52), we have the pole of the third order which leads to the contribution

$$
\frac{1}{2 \pi i} \int_{a-i \infty}^{a+i \infty} d \omega e^{\omega Y} \frac{1}{(\omega-\omega(\nu))^{2}} \frac{1}{\omega-\omega\left(\nu_{1}\right)-\omega\left(\nu_{2}\right)}=\frac{1}{2} Y^{2} e^{\omega\left(\nu_{0}\right) Y}
$$

We will not consider this contribution by the same reason that we used in calculating the 'fan' diagram since in this diagram two Pomerons with $\nu$ and $\nu^{\prime}$ turn out to be outside of the saturation region while two Pomerons with $\nu_{1}$ and $\nu_{2}$ are located inside the saturation region where we cannot use the BFKL kernel to determine the values of their intercepts. Therefore, the first 'fan' and enhanced diagrams are very similar with respect to the integration over rapidity. However, in the case of the enhanced diagram we have an additional problem: the $q^{\prime}$ integration. Indeed, even if we assume that $k>k_{0}$ and $q=0$, the integration over $q^{\prime}$ is restricted by the smallest of the two momenta $k_{1}^{\prime}$ and $k_{2}$ (see Appendix B for more details on $q^{\prime}$ integration). In other words we have the following region of integration:

1. $\quad k>k_{1}>k_{2}>k_{0}$.

In this region the first term in Eq. (A-21) contributes for all four Pomerons and we have the product of two $\delta$ functions in the vertices, namely, $\delta\left(1+\gamma-\gamma_{1}-\gamma_{2}\right) \delta\left(1+\gamma^{\prime}-\gamma_{1}-\gamma_{2}\right)$. These leads to $\gamma=\gamma^{\prime}\left(\nu=\nu^{\prime}\right)$ and the entire contribution looks the same as in the case of the first 'fan' diagram. It is worthwhile mentioning that for dipoles inside one BFKL Pomeron we have the same ordering in momenta.

2. $\quad k>k_{1}>k_{0}$ but $k_{1}<k_{2}$ while $k_{2}>k_{0}$. In this kinematic region the first Pomeron with $\nu$ and the fourth one with $\nu^{\prime}$ have contributions which stem from the first term in Eq. A-21). However, for two Pomerons with $\nu_{1}$ and $\nu_{2}$ the second terms in Eq. (A-21) play the most important roles. Collecting all factors of $k_{1}$ one can see that the integration over $k_{1}$ has the form

$$
\int d l \exp \left(\left(1-\frac{1}{2}-i \nu-i \nu_{1}-i \nu_{2}\right) l\right)
$$


where $l=\ln \left(k_{1}^{2} / k_{0}^{2}\right)$. The first term (1) in the bracket of Eq. (3.54) stemming from the integration over $q_{1} \leq k_{1}^{1}$, the second term $\left(-\frac{1}{2}-i \nu\right)$ originates from the contribution of the Pomeron with $\nu$, the third as well as the fourth terms reflects the product of the factor $\left(k_{1}^{2}\right)^{-\frac{1}{2}+i \nu_{1}}$ from the upper vertex and factor $\left(k_{1}^{2}\right)^{-2 i \nu_{1}}$ from the lower vertex since $q_{1}^{2}=k_{1}^{2}$. The integration generates $\delta\left(2-\gamma-\gamma_{1}-\gamma_{2}\right)$. The integration over $k_{2}$ has the same character as for the case of the first term and leads to $\delta\left(\gamma^{\prime}-\gamma_{1}-\gamma_{2}\right)$. These $\delta$ functions give $1-\gamma=\gamma^{\prime}$. The decomposition of Eq. (3.52) gives once more the terms shown in Fig. 3 and the pole of the third order of Eq. (3.53) since $\omega(1-\gamma)=\omega(\gamma)$ (see Eq. (3.7)). There is only one difference: in this pole of the third order, three Pomerons (with $\nu, \nu_{1}$ and $\nu_{2}$ ) are inside of the saturation region. This fact does not influence our reasoning that we need not consider this kind of contribution.

Finally, we can conclude that the first enhanced diagram has the same three contributions as the BFKL Pomeron calculus in zero transverse dimensions.

\subsection{Reduction of the emhanced diagrams to the system of non-interacting Pomerons}

In this section we will prove that an arbitrary enhanced diagram (see the diagram in Fig. 1 1 ) can be reduced to the system of non-interacting Pomerons in the kinematic region: $\alpha_{S} Y \ll 1 / \alpha_{S}$. As far as $\omega$ integration is concerned this diagram can be written as

$$
\int_{a-i \infty}^{a+i \infty} \frac{d \omega}{2 \pi i} e^{\omega Y} \gamma_{B}^{2}\left(\frac{1}{\omega-\omega(\gamma)}\right)^{m+1} \Sigma_{1}^{m}(1 P I \mid \omega)
$$

where $\gamma_{B}$ is the vertex of Pomeron interaction with the dipole ${ }^{3} \Sigma(1 P I \mid \omega)$ is the one Pomeron irreducable diagram and can be written in the following form

$$
\Sigma(1 P I \mid \omega)=-\frac{\Gamma^{2}}{\omega-\omega\left(\gamma_{1}\right)-\omega\left(\gamma_{2}\right)}+\sum_{j}(-1)^{j} \Gamma^{j} \prod_{i>2}^{j-1} \frac{1}{\omega-\sum_{l, l>2}^{i} \omega\left(\gamma_{l}\right)}
$$

In Eq. (3.55) and Eq. (3.56) $\gamma$ denotes the Pomeron - dipole vertex and $\Gamma$ is the triple Pomeron vertex (see Eq. (3.25)). The integral over $\omega$ in Eq. (3.55) can be taken closing contour over one Pomeron poles $\omega=\omega(\gamma)$ ( see the diagram in Fig. 1 2$)$ and closing contour over singularities of $\Sigma(1 P I \mid \omega)$ - the diagram in Fig. 1 3 . The first contribution has the form

$$
A(\text { Fig. 目 }-2) \propto \frac{1}{m !}\left(Y+O\left(1 / \alpha_{S}\right)\right)^{m} \exp (\omega(\gamma) Y) \Sigma_{1}^{m}(1 P I \mid \omega=\omega(\gamma))
$$

The contributions of the order of $1 / \alpha_{S}$ stem from the differentiation of the factor $\Sigma_{1}^{m}(1 P I \mid \omega)$ with respect to $\omega$ in Eq. (3.55) in taking the pole of $m+1$-order. All these contributions are so small that they can be neglected, since $Y \gg 1 / \alpha_{S}\left(\alpha_{S} Y \gg 1\right)$.

From Eq. (3.56) one can see that the first term in $\Sigma_{1}(1 P I \mid \omega)$ is of the order of $\Gamma^{2} / \omega(\gamma) \propto \alpha_{S}^{3}$ while the others lead to smaller contribution. Therefore, the only term with $m=0$ remains that gives the exchange of one Pomeron (see Fig. 1 1 -4).

The first singularity that $\Sigma_{1}(1 P I \mid \omega)$ has is the pole $\omega-\omega\left(\gamma_{1}\right)+\omega\left(\gamma_{2}\right)$. These poles are shown explicitly in Fig. $8-3$ and closing contour on these poles we obtain the contribution which is similar to Eq. (3.57), namely,

$$
A(\text { Fig. 目 }-5) \propto \frac{1}{m_{2} !} N_{2}^{2}\left(\omega=\omega\left(\gamma_{1}\right)+\omega\left(\gamma_{2}\right) /\left(Y+O\left(1 / \alpha_{S}\right)\right)^{m_{2}} \Sigma_{1}\left(2 P I \mid \omega=\omega\left(\gamma_{1}+\omega\left(\gamma_{2}\right)\right)\right.\right.
$$

\footnotetext{
${ }^{3}$ In this section we use for this vertex the notation $\gamma_{B}$ instead $\gamma$ since we reserve $\gamma$ for the anomalous dimension. We hope that in other part of the paper we use $\gamma$ for the vertex will not lead to any misunderstanding.
} 


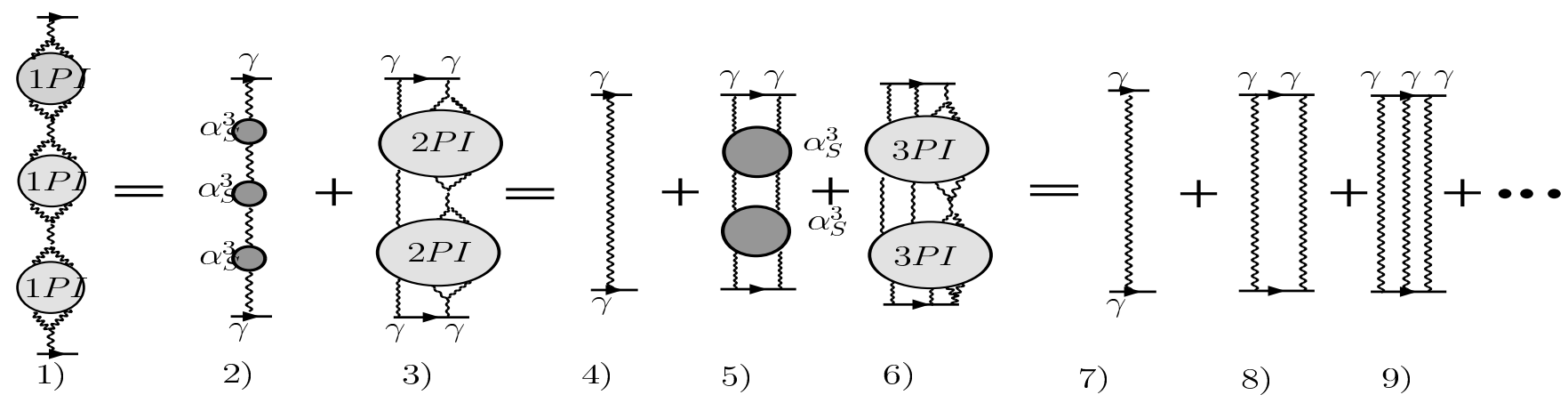

Figure 8: Reduction of the enhanced diagrams to a system of non-interecting Pomerons .

A)

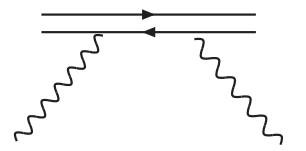

$B)$

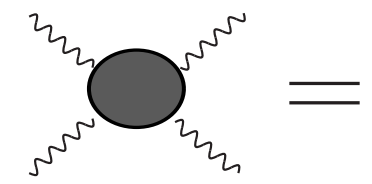

C)

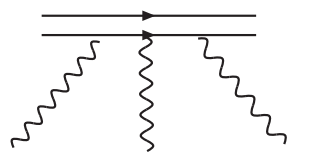

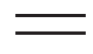

$\sum_{5}^{2}{ }^{2}$

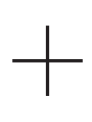

$\omega=\omega\left(\gamma_{1}\right)+\omega\left(\gamma_{2}\right)$
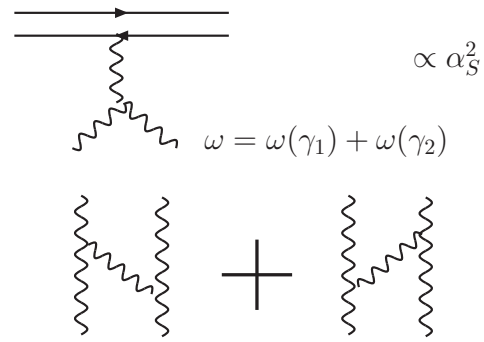

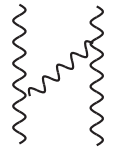

$\propto \alpha_{S}^{2}$

Figure 9: The vertices of two and three Pomerons interaction with dipoles and $\Sigma\left(2 P I \mid \omega=\omega\left(\gamma_{1}\right)+\omega\left(\gamma_{2}\right)\right)$.

where $m_{2}$ is the number of the two Pomeron poles ${ }^{4} ; N_{2}$ is the vertex of dipole-two Pomeron interaction (see Fig. 99 A) and $\Sigma_{1}(2 P I \mid \omega)$ is the sum of two Pomeron irreducable diagrams. Since the first term in $\Sigma_{1}(2 P I \mid \omega)$ gives the contribution which is proportional to $\alpha_{S}^{3}$ (see Fig. 9-B) we can neglect all contribution except with $m_{2}=0$ reducing the diagram of Fig. 8 5 to two Pomerons exchange of Fig. 8- 8 . To take the contribution from three Pomerons exchange in the diagram of Fig. \& 3 we need to consider the diagram of Fig. \& 6 and close the contour in $\omega$ over the poles $\omega=\omega\left(\gamma_{1}\right)+\omega\left(\gamma_{2}\right)+\omega\left(\gamma_{3}\right)$. Repeating the same procedure and taking into account that for the vertex of dipole-three Pomerons we have the relation shown in Fig. 9- $\mathrm{C}$ we obtain the diagram of Fig. 89. Continuing this procedure we see that we reduce the general enhanced diagram to the system of non-interacting Pomerons.

\footnotetext{
${ }^{4}$ We assume that $\gamma_{1}$ and $\gamma_{2}$ are the same for all two Pomerons poles. Actually, only sum of $\gamma_{1}+\gamma_{2}$ preserves. Strictly speaking we need to consider all two Pomerons poles a bit different and take separately each of them. It is easy to see that in doing so we get the same result as in Eq. (3.58) after integration over relevant $\gamma$ 's
} 


\section{General solution for the simplified BFKL kernel}

\subsection{The main idea}

The analysis of the first diagrams for the BFKL Pomeron calculus shows that they have the same kind of contributions as for the BFKL calculus in zero transverse dimensions and the new one that stems from the possibility that for some specific values of the anomalous dimension $\left(\gamma_{0, k}\right)$ the intercept of $n$ BFKL Pomerons in exchange is equal to the intercept of $k$ BFKL Pomerons with $k<n$. However, for this specific contribution two or more Pomerons are located inside the saturation region where we cannot use Eq. (3.7) to calculate the intercept of the Pomeron. Inside the saturation region we can use Eq. (3.47) which has a rather general proof (see Ref. [46]). In doing so we see that this specific QCD contribution leads to an energy suppressed scattering amplitude and can be neglected in the first approximation to the problem.

Having this result in mind we can restrict ourselves by calculating only terms stemming from the decomposition of the type of Eq. (3.52). This decomposition leads to the exchange of non-interacting BFKL Pomerons and to the renormalisation of the vertex for the Pomeron target (projectile) interaction as well as to the renormalisation of the Pomeron intercept. In the kinematic region given by Eq. (1.1) we can neglect the renormalisation of the Pomeron intercept and find the renormalised vertex for the Pomeron target (projectile) interaction from the initial condition to our problem since it is closely related to the dipole -dipole (or target) interaction at low energies.

Since we are dealing with the system of non-interacting Pomerons we can apply the procedure of the MPSI approximation (see Ref. [23]) to take into account all the Pomeron loops. The strategy of the improved MPSI approach consists of three major steps

1. To find the solution in the mean field approximation for arbitrary initial conditions. According to our analysis in this approximation the scattering amplitude has the following form

$$
N\left(Y-Y^{\prime},\left[\gamma_{R}\left(k_{i}, b_{i}\right)\right]\right)=\sum_{n=1}^{\infty} \int \prod_{i=1}^{n} d^{2} k_{i}(-1)^{n} C_{n}(k) P\left(k, k_{i} ; b_{i} \mid Y-Y^{\prime}\right) \gamma_{R}\left(k_{i}, b_{i}\right)
$$

where $\gamma_{R}$ is an arbitrary function of $k$ (recall that $k$ is the conjugated variable to the size of a dipole).

2. Comparing the exact solution with the initial condition to find the solution in terms of the renormalized vertices. It means that we need to solve the functional equation (in the general case)

$$
N\left(Y-Y^{\prime}=0,\left[\gamma_{R}(k, b)\right] ; b\right)=N([\gamma(k, b)])
$$

where the function $N$ should be given. In other words the scattering amplitude for low energies should be known either from calculations in QCD or from some phenomenology. This step is in full agreement with the parton model where the high energy interaction can be calculated through the low energy amplitude for the interaction of 'wee' partons that have to be given.

3. To use the MPSI formula to take into account the Pomeron loops. This formula reads as follows

$$
\begin{aligned}
& N^{I M P S I}\left(k, k_{0} ; b, Y-0\right)=\sum_{n=1}^{\infty} \frac{(-1)^{n-1}}{n !} \prod_{i=1, j=1}^{n} d^{2} k_{i} d^{2} k_{j} d^{2} b_{i} d^{2} b_{j} \frac{\delta}{\delta \gamma_{R}^{(1)}\left(k_{i}, b_{i}\right)} \frac{\delta}{\delta \gamma_{R}^{(2)}\left(k_{j}, b_{j}\right)} \\
& \left.N^{M F A}\left(Y-Y^{\prime},\left[\gamma_{R}^{(1)}\left(k_{i}, b_{i}\right)\right]\right) N^{M F A}\left(Y^{\prime},\left[\gamma_{R}^{(2)}\left(k_{j}, b_{j}\right)\right]\right)\right|_{\gamma_{R}^{(1)}=\gamma_{R}^{(2)}=0} \gamma^{B A}\left(k_{i}, k_{j}, \underline{b}-\underline{b}_{i}-\underline{b_{k}}\right)
\end{aligned}
$$


where $\gamma^{B A}$ is the amplitude in the Born approximation. It should be stressed that Eq. (4.1) for $N^{M F A}$ guarantees that $N^{I M P S I}\left(k, k_{0} ; b, Y-0\right)$ does not depend on the value of $Y^{\prime}$. Indeed, using the fact that

$$
\int d^{2} k_{i} d^{2} k_{j} d^{2} b_{i} d^{2} b_{j} P\left(k, k_{i} ; b_{i} \mid Y-Y^{\prime}\right) \gamma^{B A}\left(k_{i}, k_{j}, \underline{b}-\underline{b}_{i}-\underline{b_{k}}\right) P\left(k_{0}, k_{j} ; b_{j} \mid Y^{\prime}\right)=\bar{\alpha}_{S}^{2} P\left(k, k_{0} ; b \mid Y\right)
$$

one can rewrite Eq. (4.3) in the form

$$
\begin{aligned}
& N^{I M P S I}\left(k, k_{0} ; b, Y-0\right)=\sum_{n=1}^{\infty} \frac{(-1)^{n-1}}{n !} \prod_{i=1, j=1}^{n} d^{2} k_{i} d^{2} k_{j} d^{2} b_{i} d^{2} b_{j} \frac{\delta}{\delta \gamma_{R}^{(1)}\left(k_{i}, b_{i}\right)} \frac{\delta}{\delta \gamma_{R}^{(2)}\left(k_{j}, b_{j}\right)} \\
& \left.N^{M F A}\left(Y-Y^{\prime},\left[\gamma_{R}^{(1)}\left(k_{i}, b_{i}\right)\right]\right) N^{M F A}\left(Y^{\prime},\left[\gamma_{R}^{(2)}\left(k_{j}, b_{j}\right)\right]\right)\right|_{\gamma_{R}^{(1)}=\gamma_{R}^{(2)}=0}\left(\bar{\alpha}_{S}^{2} P\left(k, k_{0} ; b \mid Y\right)\right)^{n} \\
& =1-\left.\exp \left\{-\bar{\alpha}_{S}^{2} P\left(k, k_{0} ; b \mid Y\right) \frac{\partial}{\partial \gamma_{P}^{(1)}} \frac{\partial}{\partial \gamma_{P}^{(2)}}\right\} N^{M F A}\left(\gamma_{P}^{(1)}\right) N^{M F A}\left(\gamma_{P}^{(2)}\right)\right|_{\gamma_{P}^{(1)}=\gamma_{P}^{(2)}=0}
\end{aligned}
$$

where $\gamma_{P}^{(1)}=P\left(k, k_{i} ; b_{i} \mid Y-Y^{\prime}\right) \gamma_{R}\left(k_{i}, b_{i}\right)$ and $\gamma_{P}^{(2)}=P\left(k_{0}, k_{i} ; b_{i} \mid Y^{\prime}\right) \gamma_{R}\left(k_{i}, b_{i}\right)$

At the moment we can carry out this program only for a simplified BFKL kernel since only for this kernel do we have the exact analytical solution to work with (see [6]). The substantial number of numerical solutions [8] does not help us since we know how to perform the renormalisation of the vertices only for the analytical solution.

\subsection{The simplified BFKL kernel and MFA solution}

The kernel for which we find the solution and implement our program has the following form [6]

$$
\omega\left(\gamma=\frac{1}{2}+i \nu\right)=\bar{\alpha}_{S}\left\{\begin{aligned}
\frac{1}{\gamma} & \text { for } r^{2} Q_{s}^{2} \ll 1 ; \\
\frac{1}{1-\gamma} & \text { for } r^{2} Q_{s}^{2} \gg 1 ;
\end{aligned}\right.
$$

This kernel sums the leading log contributions in the region of low $x$. The first one is the usual LLA approximation of perturbative QCD in which the terms of the order of $\left(\alpha_{S} \ln \left(r^{2} \Lambda^{2}\right)\right)^{n}$ are taken into account for $r^{2} Q_{s}^{2} \ll 1$ where $\alpha_{S} \ln \left(r^{2} \Lambda^{2}\right) \gg 1$; and the second $\log$ approximation leads to the summation of the terms of the order of $\left(\alpha_{S} \ln \left(r^{2} Q_{s}^{2}\right)\right)^{n}$ in the kinematic region where $r^{2} Q_{s}^{2} \gg 1$ and $\alpha_{S} \ln \left(r^{2} Q_{s}^{2}\right) \gg 1$ [49, 6].

The LLA leads to the ordering of the dipole momenta.

In the perturbative QCD region $\left(r^{2} Q_{s}^{2} \ll 1\right)$ we have, using the diagrams of Fig. 7 as an example,

$$
k \gg k_{1} \gg k_{2} \gg k_{0}
$$

In the saturation region the ordering is the opposite, namely,

$$
k \ll k_{1} \ll k_{2} \ll \max \left(k_{0} \text { or } Q_{s}\right)
$$

where $Q_{s}$ is the saturation scale.

Eq. (4.6) is not only much simpler than the exact kernel of Eq. (3.7) but calculating the Pomeron diagrams in LLA we can neglect the contribution due to the overlapping of the intercept for a different number of Pomerons in exchange. Indeed, the fact that a number of Pomerons are in the saturation region while other Pomerons are outside this region, means that we cannot keep the ordering of Eq. (4.7) or Eq. (4.8). Therefore, dealing with this kernel we enhance the arguments for neglecting the overlapping singularities. 
Fortunately, the solution for the MFA has been found in Ref. [6] in the entire kinematic region for arbitrary initial conditions. For completeness of our presentation, we will describe the main features of this solution inside of the saturation region in this section. The solution is written for the amplitude in the coordinate representation, namely

$$
N\left(x_{12} ; b ; Y\right)=x_{12}^{2} \int \frac{d^{2} k}{(2 \pi)^{2}} e^{i \underline{k} \cdot \underline{x}_{12}} N(k, b ; Y)
$$

The solution in the MFA ( to the Balitsky-Kovchegov equation) can be written in the form

$$
N(z)=1-e^{-\zeta(z)}
$$

where the variable

$$
z=\ln \left(x_{12}^{2} Q_{s}^{2}(Y, b)\right)
$$

and function $-\zeta(z)$ is determined by the following equation

$$
z=\sqrt{2} \int_{\zeta_{0}(b)}^{\zeta} \frac{d \zeta^{\prime}}{\sqrt{\zeta^{\prime}+\left(\exp \left(-\zeta^{\prime}\right)-1\right)}}
$$

The boundary conditions for the solution of Eq. (4.10) we should find from the solution at $z<0$. We assume that to the right of the critical line $x_{12}^{2} Q_{s}^{2}(Y, b) \approx 1(z=0)$ the exchange of one BFKL Pomeron gives the main contribution. It has the form [7] for our simplified BFKL kernel

$$
P(z, \gamma)=\gamma e^{\frac{1}{2} z} e^{-b^{2} / R^{2}}=\gamma(b) e^{\frac{1}{2} z}
$$

where $R^{2}$ ( in the spirit of the LLA approximation ) depends only on initial conditions at low energy and has a clear non-perturbative origin. Therefore the boundary conditions have the form

$$
\begin{aligned}
N\left(z=0_{+} ; E q \cdot(4.10)\right) & =N\left(z=0_{-}\right)=\gamma e^{-b^{2} / R^{2}} \equiv \gamma(b) ; \\
\left.\frac{d \ln N}{d z}\right|_{z=0_{+}} & =\left.\frac{d \ln N}{d z}\right|_{z=0_{-}}=\frac{1}{2} ;
\end{aligned}
$$

For small $\zeta$ the solution to Eq. (4.12) has the form

$$
\ln \frac{\zeta}{\zeta_{0}(b)}=\frac{1}{2} z \text { or } \zeta=\zeta_{0}(b) e^{\frac{1}{2} z}
$$

This equation says that we can satisfy Eq. (4.14) and Eq. (4.15) if

$$
\zeta_{0}(b)=\gamma(b) \text { and } \gamma(b) \ll 1
$$

Since $\gamma(b) \approx \bar{\alpha}_{S}^{2}$ Eq. (4.17) shows that Eq. (4.12) gives the solution to our problem.

From Eq. (4.12) one can see that at small values of $\zeta_{0}$ the integral has a logarithmic divergence. Therefore, we can rewrite Eq. (4.12) as follows

$$
z+2 \ln \gamma(b)=2 \ln \left(\gamma(b) e^{\frac{1}{2} z}\right)=\sqrt{2} \int_{\zeta_{0}(b)}^{\zeta} \frac{d \zeta^{\prime}}{\sqrt{\zeta^{\prime}+\left(\exp \left(-\zeta^{\prime}\right)-1\right)}}+\ln \zeta_{0}(b)
$$

The r.h.s. of this equation does not depend on $\zeta_{0}(b)=\gamma(b)$ (see Eq. (4.17)). In Fig. 10 we plot $\zeta(z)$ for two cases : the exact expression of Eq. (4.12) and

$$
\sqrt{2} \int_{a}^{\zeta} \frac{d \zeta^{\prime}}{\sqrt{\zeta^{\prime}+\left(\exp \left(-\zeta^{\prime}\right)-1\right)}}+\ln \left(\zeta_{0}(b) / a\right)
$$


One can see that both functions coincide. This is an illustration that the integral of Eq. (4.12) has no further dependence on $\gamma(b)$. Having this in mind we can claim that Eq. (4.18) leads to a general function, namely

$$
\zeta(z)=\Phi\left(\gamma(b) e^{\frac{1}{2} z}\right) \equiv \Phi\left(\gamma_{P}(z, b)\right)
$$

The argument of the function $\Phi$ is the one Pomeron exchange (see Eq. (4.13)) and expanding $\Phi\left(\gamma(b) e^{\frac{1}{2} z}\right)$ in a series with respect to this argument we are able to find the coefficient $C_{n}$ in Eq. (4.1).

Eq. (4.12) has also two simple analytical solutions for the function $\Phi$ : in the region of small $\zeta$ ( see Eq. (4.16) and for large $\zeta$. The last solution is

$$
\zeta(z)=\frac{1}{2} \ln ^{2}\left(\gamma(b) e^{\frac{1}{2} z}\right)
$$

The exact solution is shown in Fig. 11 together with these two analytical solutions. One can see that these two analytical solutions give a good approximation for small and large values of $\gamma(b) e^{\frac{1}{2} z}$.

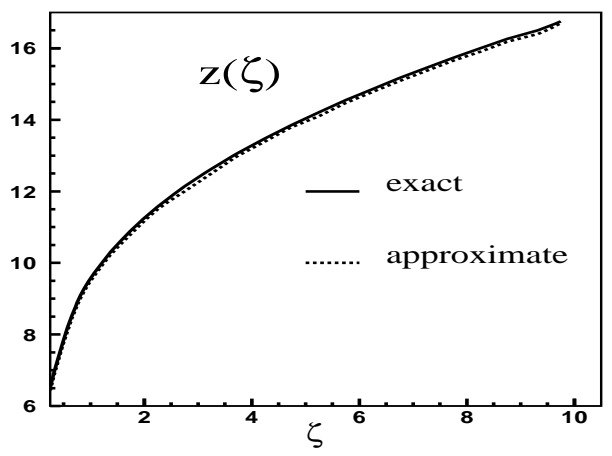

Figure 10: Comparison of the exact integral of Eq. 4.12) with the approximate formula of Eq. (4.19) with $a=0.25$.

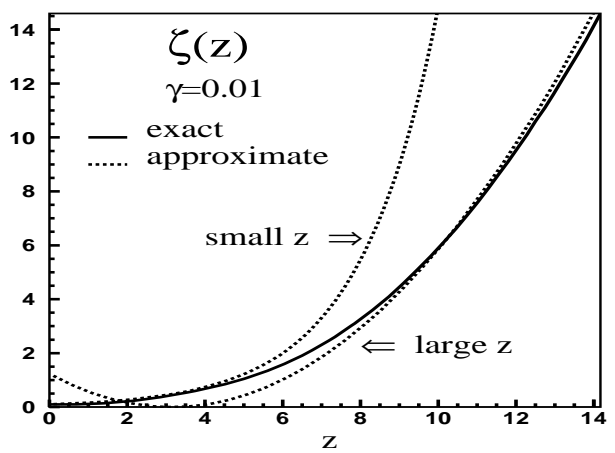

Figure 11: Solution to Eq. (4.12) (solid line) and two approximate analytical function: Eq. (4.16) (small $z$ ) and Eq. (4.21) at large $z$ (both are shown in dotted lines).

\subsection{Improved MPSI solution for Pomeron loops}

Using Eq. (4.10) and Eq. (4.20) we can find the sum of the Pomeron loops by means of Eq. (4.5). We demonstrate the answer by solving analytically two extreme cases: (i) the behaviour of the scattering amplitude near to the saturation scale; and (ii) the asymptotic behaviour deep inside of the saturation region.

For the first case $\zeta=\gamma(b) \exp \left(\frac{1}{2} z\right)$ and Eq. (4.10) together with Eq. (4.5) lead to the following answer

$$
N^{I M P S I}\left(x^{2}, R^{2} ; Y\right)=1-\exp \left(-\gamma(b) P\left(x^{2} ; R^{2} ; Y\right)\right)=1-\exp \left(-\gamma(b) e^{\frac{1}{2} z}\right)
$$

where $x$ and $R$ are the sizes of the projectile and target dipoles and $z$ is defined by Eq. (4.11) where $Q_{s}^{2} \propto 1 / R^{2}$.

For finding the behaviour of the scattering amplitude deeply inside of the saturation region we will use Eq. (2.18). In new variables: $\frac{1}{2} l-l_{1}=\ln \left(\gamma_{P}^{(1)}\right)$ and $\frac{1}{2} l+l_{1}=\ln \left(\gamma_{P}^{(2)}\right)$, the contour $C$ looks as it is shown in Fig. 12. Since $N^{M F A}$ does not have singularities in the variables $l$ and $l_{1}$ we can replace the contour $C$ by the contour $C_{R}$ (see 
Fig. 12). Therefore, we can rewrite Eq. (2.18) in the following form

$$
\begin{aligned}
N_{0}^{M P S I}(z) & =1-\frac{1}{(2 \pi i)^{2}} \oint_{C_{R}} d l \oint_{C_{R}} d l_{1} \exp \left(\frac{e^{l}}{P\left(\gamma^{B A}, z\right)}\right)\left(\frac{e^{l}}{P\left(\gamma^{B A}, z\right)}\right) \Gamma\left(0, \frac{e^{l}}{P\left(\gamma^{B A}, z\right)}\right) \\
& \times Z^{M F A}\left(P\left(e^{\frac{1}{2} l+l_{1}}\right)\right) Z^{M F A}\left(P\left(e^{\frac{1}{2} l-l_{1}}\right)\right)
\end{aligned}
$$

Using Eq. (4.13) and Eq. (4.21) we rewrite

$$
\begin{aligned}
& Z^{M F A}\left(P\left(e^{\frac{1}{2} l+l_{1}}\right)\right)=1-N^{N F A}\left(\gamma_{P}^{(1)}\right)=e^{-\zeta\left(\frac{1}{2} l+l_{1}\right)}=e^{-\frac{1}{2}\left(\frac{1}{2} l+l_{1}\right)^{2}} \\
& Z^{M F A}\left(P\left(e^{\frac{1}{2} l-l_{1}}\right)\right)=1-N^{N F A}\left(\gamma_{P}^{(2)}\right)=e^{-\zeta\left(\frac{1}{2} l-l_{1}\right)}=e^{-\frac{1}{2}\left(\frac{1}{2} l-l_{1}\right)^{2}}
\end{aligned}
$$

where $z=z_{1}+z_{2}$.

One can see that the integration over $l_{1}$ reduces to a very simple integral, namely

$$
\frac{1}{2 \pi i} \oint_{C_{R}} d l_{1} e^{-l_{1}^{2}}=\frac{1}{\pi} e^{\pi^{2}} \int_{0}^{\infty} d l_{1} \sin \left(2 \pi l_{1}\right) e^{-l_{1}^{2}}=\frac{1}{2 \sqrt{\pi}} \operatorname{erfi}(\pi)
$$

where $\operatorname{erfi}(z)=\operatorname{erf}(i z) / i$ and $\operatorname{erf}(z) \equiv E_{2}(z)=\frac{2}{\sqrt{\pi}} \int_{0}^{z}, \exp \left(-t^{2}\right) d t$ is the error function (see Refs. 40, 41]).

The remaining integral over $l$ has the form

$$
\begin{aligned}
& N_{0}^{M P S I}(z)=1 \\
& -\frac{\operatorname{erfi}(\pi)}{2 \sqrt{\pi} \pi} \int_{0}^{\infty} d l\left\{1-\exp \left(\frac{1}{\gamma^{B A} e^{\frac{1}{2} z-l}}\right)\left(\frac{1}{\gamma^{B A} e^{\frac{1}{2} z-l}}\right) \Gamma\left(0, \frac{1}{\gamma^{B A} e^{\frac{1}{2} z-l}}\right)\right\} \times \sin (2 \pi l) e^{-\frac{1}{8} l^{2}}
\end{aligned}
$$

It is clear from Eq. (4.26) that the dominant contribution stems from $l$ of the order of unity due to the exponential decrease at large $l$. Therefore, the behaviour of the integrand at large $\exp \left(\frac{1}{2} z\right)$ will dictate the resulting approach to 1 for the amplitude. At $z \gg 1$ the integrand gives $\gamma(b) \exp \left(-\frac{1}{2} z+l\right)$ behaviour. We can take the integral over $l$ which leads to the following answer:

$$
\begin{aligned}
& \frac{\operatorname{erfi}(\pi)}{2 \sqrt{\pi} \pi} \int_{0}^{\infty} d l\left\{\gamma(b) e^{\frac{1}{2} z-l}\right\} \times \sin (\pi l / 2) e^{-\frac{1}{8} l^{2}}= \\
& \frac{\operatorname{erfi}(\pi)}{2 \sqrt{\pi} \pi}\left(i e^{2} \sqrt{\frac{\pi}{2}}\left(\operatorname{erf}\left(\frac{4+i \pi}{2 \sqrt{2}}\right)+i \operatorname{erfi}\left(\frac{4+i \pi}{2 \sqrt{2}}\right)\right)\right) \frac{1}{\gamma(b) e^{\frac{1}{2} z}}
\end{aligned}
$$

The solution of Eq. (4.27) shows the geometrical scaling behaviour and at high energies $N \longrightarrow 1-\exp \left(-\frac{1}{2} z\right)$. In such a behaviour two features look unexpected: the geometrical scaling behaviour since the statistical physics motivated approach leads to a violation of this kind of behaviour [17]; and the fact that the asymptotic behaviour shows a very slow fall down in comparison with the expected $N \longrightarrow 1-\exp \left(-C z^{2}\right)$ [6, 23, 36. This slow decrease shows that the Pomeron loops essentially change the behaviour of the amplitude in the saturation region.

Our main difference to the statistical physics motivated approach is the fact that we took into account the impact parameter behaviour of the scattering amplitude. We observed that the integration in the Pomeron loops is dominated by the impact parameters of the order of the largest dipole size involved in two vertices assigned to the loop. We can face two different possibilities. In the first case the largest dipole belongs to the vertex, for which we assign our rapidity and for which the generating functional or other equation for our statistical system is written. This is the lower vertex of the Pomeron loop at the lower value of rapidity. In this case everything is in agreement with the statistical approach. In the second case the largest dipole belongs to the vertex at the top of the Pomeron 
loop at higher rapidity value which is our future in terms of the statistical approach. The future behavior of this situation cannot be described by a Markov chain without taking into account the long range rapidity correlations. It should be stressed that the appearance of the overlapping singularities cannot be included in the statistically motivated formalism until the problem is reformulated in terms of the interaction of new effective particles related to these singularities.

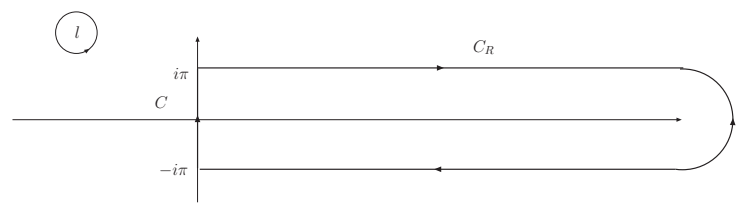

Figure 12: The contours of integration in Eq. (4.23).

\subsection{Self-consistency check}

As has been discussed we need to come back and consider the overlapping singularities that have been neglected. The check of self-consistency of our approximation means that we need to calculate all diagrams but replace the exchange of the BFKL Pomerons by the sum of all enhanced diagrams (see Fig. 13) in the kinematic region where the BFKL Pomeron enters inside of the saturation region. For example, the simplest diagram of Fig. 7 has to be replaced by the diagram of Fig. 14 in the kinematic region that is related to the overlapping singularities.

For the simplified kernel the value of $\gamma_{0}$ (see Fig. 6) is equal to 0.6 and this value is close to $\gamma_{c r}=1 / 2$. However, since $\gamma_{0}-\gamma_{c r}$ does not depend on the value of rapidity, at high energies the solution that we need to check will be at $z \gg 1$.

Indeed, the solution of Eq. (3.28) has the form of Eq. (4.13) only at small values of $\nu$. In the region of small $\nu$ the expression of Eq. (3.28) has the form

$$
\begin{aligned}
P\left(k, k_{0} \mid Y\right) & =\frac{1}{\sqrt{k^{2} k_{0}^{2}}} \int d \nu e^{\omega(\nu) Y}\left(\frac{k^{2}}{k_{0}^{2}}\right)^{i \nu} \\
& \stackrel{\nu \ll 1}{\longrightarrow} \int d \nu \exp \left(\frac{1}{2} z-i \nu z-8 \nu^{2} \bar{\alpha}_{S} Y\right) \\
& \propto \exp \left(+\frac{1}{2} z-\frac{z^{2}}{32 \bar{\alpha}_{S} Y}\right)
\end{aligned}
$$

In the last line of Eq. (4.28) we used the steepest decent method with the saddle point value of $\nu$

$$
\nu=\nu_{S P}=-i \frac{z}{16 \bar{\alpha}_{S} Y}
$$

Therefore, Eq. (4.13) for the Pomeron holds only for $z \ll 4 \sqrt{\bar{\alpha}_{S} Y}$ and at $\nu_{S P} \ll 1$. In this kinematic region $\nu_{S P} \ll 1$. In other words, the region $\gamma=\frac{1}{2} i \nu \rightarrow \gamma_{0}$ corresponds to the large values of $z$ where our scattering amplitude behaves as

$$
A \longrightarrow 1-e^{-\frac{1}{2} z}
$$

For the Pomeron near and inside of the saturation region we can rewrite the Pomeron diagrams in terms of the variables $z$ and $k$ introducing the Mellin transform for the Pomeron in the form

$$
P(z)=\frac{1}{2 \pi i} \int_{a-i \infty}^{a+i \infty} d \lambda \frac{e^{\lambda z}}{\lambda-\Delta}
$$

where the intercept $\Delta_{0}=\frac{1}{2}$. 

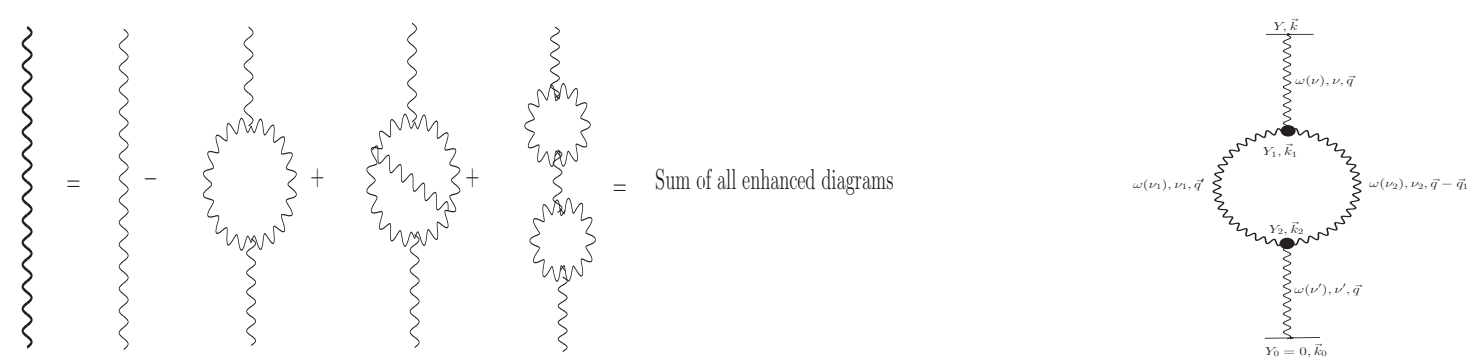

Figure 13: The graphic form for notation of wave bold line : the sum of all enhanced diagrams.

Figure 14: The diagram that we need to replace the one in Fig. 7 to study the overlapping singularities. By bold line we denote the sum of diagrams of Fig. 13 .

Eq. (4.30) has the form in $\lambda$-representation:

$$
A=\frac{1}{\lambda}-\frac{C}{\lambda+\Delta_{0}}
$$

the coefficient $C$ in Eq. (4.32) reflects the fact that we know the asymptotic behaviour inside the saturation region within the pre-exponential accuracy.

In this representation the diagram of Fig. 14 will be proportional to

$$
A\left(\text { Fig. 14) } \propto \frac{1}{2 \pi i} \int_{a-i \infty}^{a+i \infty} d \lambda e^{\lambda z} \frac{1}{\lambda-\Delta_{0}}\left\{\frac{1}{\lambda}-\frac{C}{\lambda+\Delta_{0}}\right\} \frac{1}{\lambda-\Delta_{0}}\right.
$$

instead of Eq. (3.51).

One can see that we do not have the overlapping singularities. The main contribution stems from $\lambda=\Delta_{0}$ and, since we have a double pole for this value of $\lambda$ the diagram describes the renormalisation of the Pomeron intercept which is proportional to $\bar{\alpha}_{S}^{3}$ and can be neglected in the kinematic region of Eq. (1.1).

\section{Conclusions}

The main results of this paper are:

1. In the kinematic region of Eq. (1.1) the BFKL Pomeron calculus after renormalisation of the Pomeron - target vertex, can be reduced to summing over the non-enhanced diagrams that describe the system of non-interacting Pomerons;

2. The sum of enhanced diagrams for the system of non-interacting Pomerons can be calculated by means of the improved Mueller-Patel-Salam-Iancu approach, using an additional piece of information on dipole scattering amplitude at low energy (for example, using the Born Approximation of perturbative QCD for its determination);

3. For the simplified BFKL kernel given by Eq. (4.6) the analytical solution was found to have two unexpected features: the geometrical scaling behaviour is deeply in the saturation region and is slow to approach $(N \rightarrow$ $1-C \exp \left(-\frac{1}{2} z\right)$ instead of $N \rightarrow 1-C \exp \left(-z^{2} / 8\right)$ as it happens in the MFA formalism. 
In this paper we confirm the result of Hatta and Mueller [44 that a new type of singularities appear in the BFKL Pomeron calculus: the overlapping singularities. They lead to a steeper asymptotic behaviour than the multi BFKL Pomeron exchanges give. It means that even the MFA formalism is doubtful without talking about summing over Pomeron loops. We argue that we can neglect these singularities since they stem from the kinematic region where two or more BFKL Pomerons are in the saturation region. We did the self-consistency check that such kind of singularities do not appear in the framework of the solution that we found.

As has been mentioned several times in this paper, the geometrical scaling behaviour is in contradiction with the statistical physics analogy description and the Langevin equation based on this analogy 17. However, we need to recall that the statistical-like approach at the moment exists only as a QCD motivated model since the form of the noise term that has been used, is oversimplified. Indeed, we can prove that the BFKL Pomeron calculus can be described by the Langevin equation with some noise term but this noise is so complicated ( see [50]) that we do not know how to treat it. Instead of this the simplified model for the noise term has been used. This model, as far as we understand it, has its own difficulties: (i) it cannot describe the overlapping singularities that have been discussed here; (ii) it did not include the impact parameter dependence which is very important for any treatment of the Pomeron loops as it was shown here; and (iii) even for the toy model in which we neglect the dependence of the vertices on the dipole sizes, the statistical model predicts the constant cross section at high energies ${ }^{5}$ while the exact solution (see Ref. [28] ) leads to a decrease of the total cross section.

We believe that the most vital problem, among these, is the correct impact parametre dependence. The calculation of the enhanced diagrams show that the typical impact parameter in the Pomeron loops is of the order of the smallest size of the dipoles involved in the two vertices in the loops. It might or might not be the dipole from the vertex at rapidity $\mathrm{Y}$ for which we write the equation. If this dipole belongs to the second vertex, it is related to how we proceed from here and we do not think that it is possible to describe such a system in a statistical way, assuming only short range correlations in rapidity. The failure of the toy model description by the Langevin equation is, in our opinion, due to the fact that a statistical model cannot describe the renormalization of the intercept of the Pomeron and their applicability is restricted to the kinematic region of Eq. (1.1).

Since we consider the case of fixed coupling, even in deep inelastic scttering we have to take into acount all Pomeron loops. Indeed, as it was shown in Ref. 1] only running QCD coupling selects the 'fan' diagrams and leads to the mean field approximation for these processes. One cn see that the sum of Pomeron loopsat $Q^{2}$ near to the saturation scale has a typical eikonal form (see Eq. (4.22). This result supports the numerous saturation models that describes well the experimental data [53].

However, it seems more realistically, for a description of the deep inelastic processes first to solve the problem of summation of the Pomeron loops in the case of running QCD coupling. In this case we expect [1] the the mean field approximation will be able to describe the sunstantial part of kinematic region. More that ten years ago Braun and Levin 54 suggested the procedure how to include the running QCD coupling in to th BKL equayion that describes the linear evolution in the region of high energy. This suggestion was based on the strong assumption (which by the way, were proved ) that the gluon reggeization being the essential part of the BFKL approach in the leading order will be preserved in the next-to-leading order as well. The formula that they obtained has a so called triumvirate structure, namely, for the basic process $G(\vec{q})+G\left(\vec{q}^{\prime}\right) \rightarrow G\left(\vec{q}-\vec{q}^{\prime}\right)$ the fixed coupling constand should be replaced by

$$
\alpha_{S} \rightarrow \frac{\alpha_{S}\left(\vec{q}-\vec{q}^{\prime}\right) \cdot \alpha_{S}\left(\vec{q}^{\prime}\right)}{\alpha_{S}(\vec{q})}
$$

Recently this conjucture has been proven (see Ref. [55]. This breakthrough allows us to include the running QCD coupling in the equation of the high density QCD. However, an additional problem arise that should be studied,

\footnotetext{
${ }^{5}$ We got this information from S. Munier (lecture at GGI workshop on high density QCD) and from E. Naftali (private communication).
} 
namely, such way of taking into account the running coupling effect induces the contriutions of infrared renormalons (see paper of Levin in Ref. [54] ). Such a contribution cannot be treated perturbatively and can restrict the theoretical accuracy of our approach. Therefore, summing Pomeron loops with running QCD coupling is our next challenging problem.

\section{Acknowledgments}

We want to thank Asher Gotsman, Alex Kovner, Uri Maor, Larry McLerran and Al Mueller for very useful discussions on the subject of this paper. Special thanks from A.P. goes to Carlos Pajares for his hospitality and support in the University of Santiago de Compostela where this work was completed.

This research was supported in part by the Israel Science Foundation, founded by the Israeli Academy of Science and Humanities, by a grant from Ministry of Science, Culture \& Sport, Israel \& the Russian Foundation for Basic research of the Russian Federation, and by BSF grant \# 20004019.

We thank the Galileo Galilei Institute for Theoretical Physics for the hospitality and the INFN for partial support during the completion of this work.

\section{Appendix A: Calculation of $g(\underline{k}, \underline{q}, n, \nu)$.}

We want to calculate the Fourier transform of the vertex function $E$ defined in Eq. (3.13) as

$$
g(\underline{k}, \underline{q}, n, \nu) \equiv \int \frac{d^{2} \underline{x}_{12}}{\underline{x}_{12}^{2}} d^{2} \underline{z} e^{i \underline{k} \cdot \underline{x}_{12}} e^{i q \underline{z}} E\left(\underline{x}_{1}, \underline{x}_{2} ; \underline{x}_{0} \mid n ; \nu\right)
$$

with $\underline{z}=\left(\underline{x}_{1}+\underline{x}_{2}\right) / 2-\underline{x}_{0}$. The similar Fourier transform was already calculated in [52], but the authors used a different normalization and we found it useful to calculate it using our notation. The integration over $\underline{z}$ was done by Navelet and Peschanski in [43] by considering the solutions of the differential equations obeyed by the vertex functions $E$ and consequent matching of the result to an approximate one obtained by Lipatov in [42]. We adopt the notation introduced by Lipatov for the mixed representation of the vertex functions

$$
E_{q}^{n, \nu}(\rho)=\frac{2 \pi^{2}}{b_{n, \nu}} \frac{1}{|\rho|} \int d z d \bar{z} e^{\frac{i}{2}(\bar{q} z+q \bar{z})} E^{n, \nu}(z+\rho / 2, z-\rho / 2)
$$

where

$$
b_{n, \nu}=\frac{2^{4 i \nu} \pi^{3}}{|n| / 2-i \nu} \frac{\Gamma(|n| / 2-i \nu+1 / 2) \Gamma(|n| / 2+i \nu)}{\Gamma(|n| / 2+i \nu+1 / 2) \Gamma(|n| / 2-i \nu)}
$$

and $\rho=x_{12}$ in our notation introduced in Eq. (3.6). The expression for $E_{q}^{n, \nu}(\rho)$ is given in [43] and reads

$$
\begin{aligned}
E_{q}^{n, \nu}(\rho)= & \bar{q}^{i \nu-n / 2} q^{i \nu+n / 2} 2^{-6 i \nu} \Gamma(1-i \nu+|n| / 2) \Gamma(1-i \nu-|n| / 2) \times \\
& {\left[J_{n / 2-i \nu}\left(\frac{\bar{q} \rho}{4}\right) J_{-n / 2-i \nu}\left(\frac{q \bar{\rho}}{4}\right)-(-1)^{n} J_{-n / 2+i \nu}\left(\frac{\bar{q} \rho}{4}\right) J_{n / 2+i \nu}\left(\frac{\bar{q} \rho}{4}\right)\right] }
\end{aligned}
$$

Thus in terms of $E_{q}^{n, \nu}(\rho)$ the Fourier transform in Eq. A-1) is given by 


$$
g(\underline{k}, \underline{q}, n, \nu)=\frac{b_{n, \nu}}{2 \pi^{2}} \int \frac{d^{2} \underline{\rho}}{|\underline{\rho}|} e^{i \rho} \underline{\underline{k}} \underline{k} E_{q}^{n, \nu}(\rho)
$$

From Eq. (A-4) and Eq. (A-5) one can see that the calculation of $g(\underline{k}, \underline{q}, n, \nu)$ is reduced to evaluation of the following integral

$$
\mathcal{I}_{\mu, \mu^{\prime}}(q, k) \equiv \int \frac{d^{2} \underline{\rho}}{|\underline{\rho}|} e^{i \underline{\rho} \cdot \underline{k}} J_{\mu}\left(\frac{\bar{q} \rho}{4}\right) J_{\mu^{\prime}}\left(\frac{q \bar{\rho}}{4}\right)
$$

We introduce polar coordinates for complex variables

$$
q=|q| e^{i \psi} \quad \quad \quad \quad k=|\rho| e^{i \phi} \quad k \mid e^{i \theta}
$$

and rewrite Eq. (A-6) as

$$
\mathcal{I}_{\mu, \mu^{\prime}}(q, k) \equiv \int_{0}^{\infty} \frac{\rho d \rho}{\rho} \int_{0}^{2 \pi} d \phi e^{i \rho k \cos (\phi-\theta)} J_{\mu}\left(\frac{q \rho}{4} e^{i(\phi-\psi)}\right) J_{\mu^{\prime}}\left(\frac{q \rho}{4} e^{-i(\phi-\psi)}\right)
$$

For the sake of simplicity in Eq. (A-8) and further below we denote by $\rho, q$ and $k$ their respective absolute values. We use the series representation for the Bessel functions

$$
J_{\kappa}(z)=\left(\frac{z}{2}\right)^{\kappa} \sum_{m=0}^{\infty} \frac{(-1)^{m}}{m ! \Gamma(\kappa+m+1)}\left(\frac{z}{2}\right)^{2 m}
$$

to recast Eq. (A-8) into

$$
\begin{aligned}
\mathcal{I}_{\mu, \mu^{\prime}}(q, k)= & \int_{0}^{\infty} d \rho \int_{0}^{2 \pi} d \phi e^{i \rho k \cos (\phi-\theta)} \times \\
& e^{i \mu(\phi-\psi)}\left(\frac{q \rho}{8}\right)^{\mu} \sum_{m=0}^{\infty} \frac{(-1)^{m}}{m ! \Gamma(\mu+m+1)}\left(\frac{q \rho}{8}\right)^{2 m} e^{i 2 m(\phi-\psi)} \times \\
& e^{-i \mu^{\prime}(\phi-\psi)}\left(\frac{q \rho}{8}\right)^{\mu^{\prime}} \sum_{m^{\prime}=0}^{\infty} \frac{(-1)^{m^{\prime}}}{m^{\prime} ! \Gamma\left(\mu^{\prime}+m^{\prime}+1\right)}\left(\frac{q \rho}{8}\right)^{2 m^{\prime}} e^{-i 2 m^{\prime}(\phi-\psi)}
\end{aligned}
$$

Changing variables $\phi-\theta \rightarrow \phi$ and using the integral representation of the Bessel functions

$$
\begin{gathered}
J_{n}(z)=\frac{1}{2 \pi} \int_{-\pi}^{\pi} e^{-i n \theta+i z \sin \theta} d \theta=\frac{1}{2 \pi} \int_{-\pi-\pi / 2}^{\pi-\pi / 2} e^{-i n(\theta-\pi / 2)+i z \sin (\theta-\pi / 2)} d(\theta-\pi / 2) \\
=\frac{(-i)^{n}}{2 \pi} \int_{-3 \pi / 2}^{\pi / 2} e^{-i n \theta+i z \cos \theta} d \theta=\frac{(-i)^{n}}{2 \pi} \int_{0}^{2 \pi} e^{-i n \theta+i z \cos \theta} d \theta
\end{gathered}
$$

we rewrite Eq. (A-10) as

$$
\begin{aligned}
\mathcal{I}_{\mu, \mu^{\prime}}(q, k)=\sum_{m=0}^{\infty} \sum_{m^{\prime}=0}^{\infty} \int_{0}^{\infty} d \rho & (2 \pi) J_{-\mu-2 m+\mu^{\prime}+2 m}(\rho k) \times \\
& i^{-\mu} e^{i \mu(\theta-\psi)}\left(\frac{q \rho}{8}\right)^{\mu} \frac{1}{m ! \Gamma(\mu+m+1)}\left(\frac{q \rho}{8}\right)^{2 m} e^{i 2 m(\theta-\psi)} \times \\
& i^{\mu^{\prime}} e^{-i \mu^{\prime}(\theta-\psi)}\left(\frac{q \rho}{8}\right)^{\mu^{\prime}} \frac{1}{m^{\prime} ! \Gamma\left(\mu^{\prime}+m^{\prime}+1\right)}\left(\frac{q \rho}{8}\right)^{2 m^{\prime}} e^{-i 2 m^{\prime}(\theta-\psi)}
\end{aligned}
$$


Now we perform the integration over $\rho$ using the formula

$$
\int_{0}^{\infty} d \rho J_{\alpha}(\rho k)\left(\frac{\rho q}{8}\right)^{\beta}=\frac{1}{k}\left(\frac{q}{4 k}\right)^{\beta} \frac{\Gamma\left(\frac{1}{2}+\frac{\alpha+\beta}{2}\right)}{\Gamma\left(\frac{1}{2}+\frac{\alpha-\beta}{2}\right)}
$$

then the expression in Eq. (A-12) reads

$$
\begin{aligned}
\mathcal{I}_{\mu, \mu^{\prime}}(q, k)=\frac{2 \pi}{k} & i^{-\mu} e^{i \mu(\theta-\psi)}\left(\frac{q}{4 k}\right)^{\mu} \sum_{m=0}^{\infty} \frac{1}{m ! \Gamma(\mu+m+1)}\left(\frac{q}{4 k}\right)^{2 m} e^{i 2 m(\theta-\psi)} \frac{1}{\Gamma(1 / 2-\mu-2 m)} \times \\
& i^{\mu^{\prime}} e^{-i \mu^{\prime}(\theta-\psi)}\left(\frac{q}{4 k}\right)^{\mu^{\prime}} \sum_{m^{\prime}=0}^{\infty} \frac{1}{m^{\prime} ! \Gamma\left(\mu^{\prime}+m^{\prime}+1\right)}\left(\frac{q}{4 k}\right)^{2 m^{\prime}} e^{-i 2 m^{\prime}(\theta-\psi)} \Gamma\left(1 / 2+\mu^{\prime}+2 m^{\prime}\right)
\end{aligned}
$$

One should note the full factorization of two series in Eq. (A-14) due to the integration over $\rho$. This allows us to sum them separately using

$$
\sum_{m=0}^{\infty} \frac{z^{2 m}}{m ! \Gamma(\mu+m+1)} \frac{1}{\Gamma(1 / 2-\mu-2 m)}=\frac{\cos (\pi \mu)}{\pi} \frac{\Gamma(1 / 2+\mu)}{\Gamma(1+\mu)}{ }_{2} F_{1}\left[1 / 4+\mu / 2,3 / 4+\mu / 2 ; 1+\mu ; 4 z^{2}\right]
$$

and

$$
\sum_{m=0}^{\infty} \frac{z^{2 m}}{m ! \Gamma\left(\mu^{\prime}+m+1\right)} \Gamma\left(1 / 2+\mu^{\prime}+2 m\right)=\frac{\Gamma\left(1 / 2+\mu^{\prime}\right)}{\Gamma\left(1+\mu^{\prime}\right)}{ }_{2} F_{1}\left[1 / 4+\mu^{\prime} / 2,3 / 4+\mu^{\prime} / 2 ; 1+\mu^{\prime} ; 4 z^{2}\right]
$$

With help of Eq. $\mathrm{A}-15)$ and Eq. $(\mathrm{A}-16)$ we obtain the final expression for $\mathcal{I}_{\mu, \mu^{\prime}}(q, k)$ as

$$
\begin{aligned}
\mathcal{I}_{\mu, \mu^{\prime}}(q, k)=\frac{2 \pi}{k} \quad & i^{-\mu} e^{i \mu(\theta-\psi)}\left(\frac{q}{4 k}\right)^{\mu} \frac{\cos (\pi \mu)}{\pi} \frac{\Gamma(1 / 2+\mu)}{\Gamma(1+\mu)}{ }_{2} F_{1}\left[1 / 4+\mu / 2,3 / 4+\mu / 2 ; 1+\mu ; 4\left(\frac{q}{4 k}\right)^{2} e^{i 2(\theta-\psi)}\right] \times \\
& i^{\mu^{\prime}} e^{-i \mu^{\prime}(\theta-\psi)}\left(\frac{q}{4 k}\right)^{\mu^{\prime}} \frac{\Gamma\left(1 / 2+\mu^{\prime}\right)}{\Gamma\left(1+\mu^{\prime}\right)}{ }_{2} F_{1}\left[1 / 4+\mu^{\prime} / 2,3 / 4+\mu^{\prime} / 2 ; 1+\mu^{\prime} ; 4\left(\frac{q}{4 k}\right)^{2} e^{-i 2(\theta-\psi)}\right]
\end{aligned}
$$

At this point we return to complex vector notation given in Eq. (A-7) and absorb the angles into complex vectors $q$, $k, \bar{q}$ and $\bar{k}$

$$
\begin{gathered}
\mathcal{I}_{\mu, \mu^{\prime}}(q, k)=\frac{2 \pi}{|k|} \quad i^{-\mu}\left(\frac{\bar{q}}{4 \bar{k}}\right)^{\mu} \frac{1}{\Gamma(1+\mu) \Gamma(1 / 2-\mu)}{ }_{2} F_{1}\left[1 / 4+\mu / 2,3 / 4+\mu / 2 ; 1+\mu ;\left(\frac{\bar{q}}{2 \bar{k}}\right)^{2}\right] \times \\
i^{\mu^{\prime}}\left(\frac{q}{4 k}\right)^{\mu^{\prime}} \frac{\Gamma\left(1 / 2+\mu^{\prime}\right)}{\Gamma\left(1+\mu^{\prime}\right)}{ }_{2} F_{1}\left[1 / 4+\mu^{\prime} / 2,3 / 4+\mu^{\prime} / 2 ; 1+\mu^{\prime} ;\left(\frac{q}{2 k}\right)^{2}\right]
\end{gathered}
$$

In the first line in Eq. (A-18) we used identity $\Gamma(1 / 2+z) \Gamma(1 / 2-z)=\cos (z \pi) / \pi$. Plugging Eq. (A-18) into Eq. (A-5) we find the Fourier transform of the vertex functions $E$ defined in Eq. (A-1)

$$
\begin{aligned}
g(\underline{k}, \underline{q}, n, \nu)= & \frac{b_{n, \nu}}{2 \pi^{2}} i^{n} \bar{q}^{i \nu-n / 2} q^{i \nu+n / 2} 2^{-6 i \nu} \Gamma(1-i \nu+|n| / 2) \Gamma(1-i \nu-|n| / 2) \times \\
& {\left[\mathcal{I}_{n / 2-i \nu,-n / 2-i \nu}(q, k)-(-1)^{n} \mathcal{I}_{-n / 2+i \nu, n / 2+i \nu}(q, k)\right] }
\end{aligned}
$$

with $b_{n, \nu}$ given in Eq. (A-3). In the high energy limit the main contribution to Pomeron Green function comes from $n=0$. We are interested in the case of small momentum transferred along the Pomeron which implies

$$
|k| \gg|q|
$$

In the limit of Eq. (A-20) for $n=0$ Eq. (A-19) reads

$$
\begin{aligned}
g(\underline{k}, \underline{q}, 0, \nu)= & \frac{b_{0, \nu}}{2 \pi^{2}}|q|^{2 i \nu} 2^{-6 i \nu} \Gamma^{2}(1-i \nu) \cos (i \nu \pi)\left[\mathcal{I}_{-i \nu,-i \nu}(q, k)-\mathcal{I}_{+i \nu,+i \nu}(q, k)\right]= \\
& \simeq \frac{\pi^{2}}{-i \nu} 2^{2 i \nu}|k|^{-1+2 i \nu} \frac{\Gamma^{2}(1 / 2-i \nu) \Gamma(i \nu)}{\Gamma^{2}(1 / 2+i \nu) \Gamma(-i \nu)}-\frac{\pi^{2}}{-i \nu} 2^{-6 i \nu}|k|^{-1-2 i \nu}|q|^{4 i \nu} \frac{\Gamma^{2}(1-i \nu) \Gamma(i \nu)}{\Gamma^{2}(1+i \nu) \Gamma(-i \nu)}
\end{aligned}
$$




\section{Appendix B: Triple Pomeron vertex in $q$ and $\nu$ representation.}

In this appendix we calculate the triple Pomeron vertex in $q$ and $\nu$ representation, namely,

$$
\Gamma_{3 P}\left(q_{1}^{\prime}, q_{2}^{\prime} ; \nu_{1}, \nu_{2}, \nu_{3}\right) \equiv \int d^{2} k_{1}^{\prime} g\left(\underline{k}_{1}^{\prime}, \underline{q}_{1}^{\prime}, 0, \nu_{1}\right) g^{*}\left(\underline{k}_{1}^{\prime}+\frac{1}{2} \underline{q}_{2}^{\prime}, \underline{q}_{2}^{\prime}, 0, \nu_{2}\right) g^{*}\left(\underline{k}_{1}^{\prime}-\underline{q}_{1}^{\prime}+\frac{1}{2} \underline{q}_{2}^{\prime}, \underline{q}_{1}^{\prime}-\underline{q}_{2}^{\prime}, 0, \nu_{3}\right)
$$

where $g$ is given by Eq. (A-21).

Introducing new holomorphic variables

$$
t=\frac{q_{2}^{\prime}}{2 k_{1}^{\prime}+q_{2}^{\prime}} \quad \text { and } \quad \bar{t}=\frac{\bar{q}_{2}^{\prime}}{2 \bar{k}_{1}^{\prime}+\bar{q}_{2}^{\prime}}
$$

and using Eq. $(\mathrm{A}-18)$, we can rewrite Eq. (B-1) in the form

$$
\begin{aligned}
& \Gamma_{3 P}\left(0, q_{2}^{\prime} ; \nu, \nu_{2}, \nu_{3}\right)=4\left(\frac{q_{2}^{\prime 2}}{4}\right)^{1 / 2-i \nu_{1}+i \nu_{2}+i \nu_{3}} \int d t d \bar{t}((1-t)(1-\bar{t}))^{1 / 2+i \nu_{1}} C_{1}\left(\nu_{1}\right) \\
& \times\left\{C_{1}^{*}\left(\nu_{2}\right)_{2} F_{1}\left(1 / 4+i \nu_{2} / 2,3 / 4+i \nu_{2} / 2,1+i \nu_{2}, t^{2}\right){ }_{2} F_{1}\left(1 / 4+i \nu_{2} / 2,3 / 4+i \nu_{2} / 2,1+i \nu_{2}, \bar{t}^{2}\right)\right. \\
& \left.-C_{2}^{*}\left(\nu_{2}\right)(4 t \bar{t})^{2 i \nu_{2}}{ }_{2} F_{1}\left(1 / 4+i \nu_{2} / 2,3 / 4+i \nu_{2} / 2,1+i \nu_{2}, t^{2}\right){ }_{2} F_{1}\left(1 / 4+i \nu_{2} / 2,3 / 4+i \nu_{2} / 2,1+i \nu_{2}, \bar{t}^{2}\right)\right\} \\
& \times\left\{C_{1}^{*}\left(\nu_{3}\right)_{2} F_{1}\left(1 / 4+i \nu_{3} / 2,3 / 4+i \nu_{3} / 2,1+i \nu_{3}, t^{2}\right){ }_{2} F_{1}\left(1 / 4+i \nu_{3} / 2,3 / 4+i \nu_{3} / 2,1+i \nu_{3}, \bar{t}^{2}\right)\right. \\
& \left.-C_{2}^{*}\left(\nu_{3}\right)(4 t \bar{t})_{2}^{2 i \nu_{3}}{ }_{2} F_{1}\left(1 / 4+i \nu_{3} / 2,3 / 4+i \nu_{3} / 2,1+i \nu_{3}, t^{2}\right){ }_{2} F_{1}\left(1 / 4+i \nu_{3} / 2,3 / 4+i \nu_{3} / 2,1+i \nu_{3}, \bar{t}^{2}\right)\right\}
\end{aligned}
$$

with

$$
C_{1}(\nu)=\frac{\pi^{2}}{-i \nu} 2^{2 i \nu} \frac{\Gamma^{2}(1 / 2-i \nu) \Gamma(i \nu)}{\Gamma^{2}(1 / 2+i \nu) \Gamma(-i \nu)} ; \quad C_{2}(\nu)=\frac{\pi^{2}}{-i \nu} 2^{-6 i \nu} \frac{\Gamma^{2}(1-i \nu) \Gamma(i \nu)}{\Gamma^{2}(1+i \nu) \Gamma(-i \nu)}
$$

For simplicity we consider $\Gamma_{3 P}\left(q_{1}^{\prime}, q_{2}^{\prime} ; \nu_{1}, \nu_{2}, \nu_{3}\right)$ in Eq. (B-3) for $q_{1}^{\prime}=0$.

The integral of Eq. (B-4) has three region of potential divergency: $t \rightarrow 0, t \rightarrow 1$ and $t \rightarrow \infty$. First, let us study the behaviour of the integrant at $t \rightarrow \infty$. For doing this we use ( see formula 9.132(2) of Ref. 40]) the following expression

$$
\begin{aligned}
{ }_{2} F_{1}\left(1 / 4+i \nu_{2} / 2,3 / 4+i \nu_{2} / 2,1+i \nu_{2}, t^{2}\right) & =\frac{\Gamma\left(1+i \nu_{2}\right) \Gamma(1 / 2)}{\Gamma^{2}\left(3 / 4+i \nu_{2}\right)}\left(-\frac{1}{t^{2}}\right)_{2}^{\frac{1}{4}+i \frac{\nu_{2}}{2}}{ }_{2} F_{1}\left(1 / 4+i \nu_{2} / 2,1 / 4+i \nu_{2} / 2, \frac{1}{t^{2}}\right)(\mathrm{B}-5) \\
& +\frac{\Gamma\left(1+i \nu_{2}\right) \Gamma(-1 / 2)}{\Gamma^{2}\left(1 / 4+i \nu_{2} / 2\right)}\left(-\frac{1}{t^{2}}\right)^{\frac{3}{4}+i \frac{\nu_{2}}{2}}{ }_{2} F_{1}\left(3 / 4+i \nu_{2} / 2,3 / 4+i \nu_{2} / 2, \frac{1}{t^{2}}\right)
\end{aligned}
$$

From Eq. (B-5) we conclude that the integrant of Eq. (B-3) decreases at large $t$ as $t^{-2}$ for $\nu_{2}=\nu_{3}=0$. It means that integrals over $t$ and $\bar{t}$ are convergent as far as large $t$ and $\bar{t}$ are concerned. The next suspicious region is $t \rightarrow 0$ $(\bar{t} \rightarrow 0)$. Factor $(t \bar{t})^{-1 / 2+i \nu_{1}-i \nu_{2}-i \nu_{3}}$ in the integrant indicates a possible divergence in this region. Rewriting the integral in a general form

$$
\begin{aligned}
& \Gamma_{3 P}\left(0, q_{2}^{\prime} ; \nu_{1}, \nu_{2}, \nu_{3}\right)=\int_{0}^{\infty} \pi d(t \bar{t})(t \bar{t})^{-1 / 2+i \nu_{1}-i \nu_{2}-i \nu_{3}} \Phi(t, \bar{t}) \\
& =\int_{0}^{1} d(t \bar{t})(t \bar{t})^{-1 / 2+i \nu_{1}-i \nu_{2}-i \nu_{3}}\{\Phi(0)+(\Phi(t, \bar{t})-\Phi(0))\}+\int_{1}^{\infty} d(t \bar{t})(t \bar{t})^{-1 / 2+i \nu_{1}-i \nu_{2}-i \nu_{3}} \Phi(t, \bar{t})
\end{aligned}
$$


we see that if $\Phi(t, \bar{t})-\Phi(0) \propto t \bar{t}$ at $t \rightarrow 0$ we have a pole $1 /\left(1 / 2+i \nu_{1}-i \nu_{2}-i \nu_{3}\right)$. However, since our function $\Phi(t, \bar{t})$ has factors $t^{1 / 2+i \nu_{i}}$ we need to be more careful. In calculating first enhanced diagram (see section 3.4) $\nu_{2}$ and $\nu_{3}$ are small and $\Phi(t, \bar{t})$ has the following form

$$
\Phi(t, \bar{t})=C_{1}\left(\nu_{1}\right) \frac{\pi^{4}}{-\nu_{2} \nu_{3}}\left(1-(4 t \bar{t})^{2 i \nu_{2}}\right) \times\left(1-(4 t \bar{t})^{2 i \nu_{3}}\right) \stackrel{\nu_{2} \rightarrow 0, \nu_{3} \rightarrow 0}{\longrightarrow} C_{1}\left(\nu_{1}\right) 4 \pi^{4} \ln ^{2}(4 t \bar{t})
$$

Using Eq. (B-7) we obtain that

$$
\Gamma_{3 P}\left(0, q_{2}^{\prime} ; \nu_{1}, \nu_{2}, \nu_{3}\right) \stackrel{\nu_{2} \rightarrow 0, \nu_{3} \rightarrow 0}{\longrightarrow} \frac{C_{1}\left(\nu_{1}\right) 4 \pi^{4}\left(\frac{q_{2}^{\prime 2}}{4}\right)^{1 / 2-i \nu_{1}+i \nu_{2}+i \nu_{3}}}{\left(1 / 2+i \nu_{1}-i \nu_{2}-i \nu_{3}\right)^{3}}+\text { function without singularities in } \nu_{2} \text { and } \nu_{3}
$$

At $t \bar{t} \rightarrow 1$ we could have a divergence which leads to a pole $1 /\left(3 / 2+i \nu_{1}\right)$. However, since $C_{1}\left(\nu_{1}\right)$ has a zero of the second order at $i \nu_{1}=-3 / 2 \Gamma_{3 P}\left(0, q_{2}^{\prime} ; \nu_{1}, \nu_{2}, \nu_{3}\right)$ has no such singularity. Therefore, Eq. (B-7) gives the triple Pomeron at small $\nu_{2}$ and $\nu_{3}$. One can see that the pole in Eq. (B-8) replaces the $\delta$-function which we obtained integrating first of $q_{2}^{\prime}$.

For arbitrary $\nu_{2}$ and $\nu_{3}$ the triple Pomeron vertex has a more complicated form, namely,

$$
\begin{aligned}
\Gamma_{3 P}\left(0, q_{2}^{\prime} ; \nu_{1}, \nu_{2}, \nu_{3}\right) & =C_{1}\left(\nu_{1}\right) 4\left(\frac{q_{2}^{\prime 2}}{4}\right)^{1 / 2-i \nu_{1}+i \nu_{2}+i \nu_{3}} \times\left\{\frac{C_{1}^{*}\left(\nu_{2}\right) C_{1}^{*}\left(\nu_{3}\right)}{1 / 2+i \nu_{1}-i \nu_{2}-i \nu_{3}}-\frac{C_{1}^{*}\left(\nu_{2}\right) C_{2}^{*}\left(\nu_{3}\right)}{1 / 2+i \nu_{1}-i \nu_{2}+i \nu_{3}}(\mathrm{~B}-9)\right. \\
& \left.-\frac{C_{2}^{*}\left(\nu_{2}\right) C_{1}^{*}\left(\nu_{3}\right)}{1 / 2+i \nu_{1}+i \nu_{2}-i \nu_{3}}+\frac{C_{2}^{*}\left(\nu_{2}\right) C_{2}^{*}\left(\nu_{3}\right)}{1 / 2+i \nu_{1}+i \nu_{2}+i \nu_{3}}\right\}+ \text { regular function of } \nu_{2} \text { and } \nu_{3}
\end{aligned}
$$

Integration over $q_{2}^{\prime}$ leads $\delta\left(\nu-\nu^{\prime}\right)$ where $\nu\left(\nu_{2}\right.$ in Eq. (3.49)) corresponds to upper BFKL Pomeron in Fig. 7 , and $\nu^{\prime}\left(\nu_{4}\right.$ in Eq. (3.49) ) corresponds to lower BFKL Pomeron in Fig. ㄱ.

\section{References}

[1] L. V. Gribov, E. M. Levin and M. G. Ryskin, Phys. Rep. 100, 1 (1983).

[2] A. H. Mueller and J. Qiu, Nucl. Phys.,427 B 268 (1986) .

[3] L. McLerran and R. Venugopalan, Phys. Rev. D 49,2233, 3352 (1994); D 50,2225 (1994); D 53,458 (1996); D 59,09400 (1999).

[4] I. Balitsky, [arXiv:hep-ph/9509348]; Phys. Rev. D60, 014020 (1999) [arXiv:hep-ph/9812311] Y. V. Kovchegov, Phys. Rev. D60, 034008 (1999), [arXiv:hep-ph/9901281].

[5] J. Jalilian-Marian, A. Kovner, A. Leonidov and H. Weigert, Phys. Rev. D59, 014014 (1999), [arXiv:hep-ph/9706377]; Nucl. Phys. B504, 415 (1997), [arXiv:hep-ph/9701284]; J. Jalilian-Marian, A. Kovner and H. Weigert, Phys. Rev. D59, 014015 (1999), [arXiv:hep-ph/9709432]; A. Kovner, J. G. Milhano and H. Weigert, Phys. Rev. D62, 114005 (2000), [arXiv:hep-ph/0004014]; E. Iancu, A. Leonidov and L. D. McLerran, Phys. Lett. B510, 133 (2001); [arXiv:hep-ph/0102009]; Nucl. Phys. A692, 583 (2001), [arXiv:hep-ph/0011241]; E. Ferreiro, E. Iancu, A. Leonidov and L. McLerran, Nucl. Phys. A703, 489 (2002), [arXiv:hep-ph/0109115]; H. Weigert, Nucl. Phys. A703, 823 (2002), [arXiv:hep-ph/0004044].

[6] E. Levin and K. Tuchin, Nucl. Phys. A693 (2001) 787 [arXiv:hep-ph/0101275]; A691 (2001) 779 [arXiv:hep-ph/0012167]; B573 (2000) 833 [arXiv:hep-ph/9908317].

[7] E. Iancu, K. Itakura and L. McLerran, Nucl. Phys. A708 (2002) 327 [arXiv:hep-ph/0203137]. 
[8] N. Armesto and M. A. Braun, Eur. Phys. J. C20, 517 (2001) [arXiv:hep-ph/0104038]; M. Lublinsky, Eur. Phys. J. C21, 513 (2001) [arXiv:hep-ph/0106112]; E. Levin and M. Lublinsky, Nucl. Phys. A712, 95 (2002) [arXiv:hep-ph/0207374]; Nucl. Phys. A712, 95 (2002) [arXiv:hep-ph/0207374]; Eur. Phys. J. C22, 647 (2002) [arXiv:hep-ph/0108239]; M. Lublinsky, E. Gotsman, E. Levin and U. Maor, Nucl. Phys. A696, 851 (2001) [arXiv:hep-ph/0102321]; Eur. Phys. J. C27, 411 (2003) [arXiv:hep-ph/0209074]; K. Golec-Biernat, L. Motyka and A.Stasto, Phys. Rev. D65, 074037 (2002) [arXiv:hep-ph/0110325]; E. Iancu, K. Itakura and S. Munier, Phys. Lett. B590 (2004) 199 [arXiv:hep-ph/0310338]. K. Rummukainen and H. Weigert, Nucl. Phys. A739, 183 (2004) [arXiv:hep-ph/0309306]; K. Golec-Biernat and A. M. Stasto, Nucl. Phys. B668, 345 (2003)

[arXiv:hep-ph/0306279]; E. Gotsman, M. Kozlov, E. Levin, U. Maor and E. Naftali, Nucl. Phys. A742, 55 (2004) [arXiv:hep-ph/0401021]; K. Kutak and A. M. Stasto, Eur. Phys. J. C41, 343 (2005) [arXiv:hep-ph/0408117]; G. Chachamis, M. Lublinsky and A. Sabio Vera, Nucl. Phys. A748, 649 (2005) [arXiv:hep-ph/0408333]; J. L. Albacete, N. Armesto, J. G. Milhano, C. A. Salgado and U. A. Wiedemann, Phys. Rev. D71, 014003 (2005) [arXiv:hep-ph/0408216]; E. Gotsman, E. Levin, U. Maor and E. Naftali, Nucl. Phys. A750 (2005) 391 [arXiv:hep-ph/0411242].

[9] A. H. Mueller, A. I. Shoshi and S. M. H. Wong, Nucl. Phys. B 715, 440 (2005) [arXiv:hep-ph/0501088].

[10] E. Levin and M. Lublinsky, Nucl. Phys. A 763, 172 (2005) [arXiv:hep-ph/0501173].

[11] E. Iancu and D. N. Triantafyllopoulos, Nucl. Phys. A756, 419 (2005) [arXiv:hep-ph/0411405]; Phys. Lett. B610, 253 (2005) [arXiv:hep-ph/0501193].

[12] A. Kovner and M. Lublinsky, Phys. Rev. D 71, 085004 (2005) [arXiv:hep-ph/0501198].

[13] Y. Hatta, E. Iancu, L. McLerran, A. Stasto and D. N. Triantafyllopoulos, Nucl. Phys. A 764, 423 (2006) [arXiv:hep-ph/0504182].

[14] E. A. Kuraev, L. N. Lipatov, and F. S. Fadin, Sov. Phys. JETP 45, 199 (1977); Ya. Ya. Balitsky and L. N. Lipatov, Sov. J. Nucl. Phys. 28, 22 (1978).

[15] J. Bartels, M. Braun and G. P. Vacca, Eur. Phys. J. C40, 419 (2005) [arXiv:hep-ph/0412218] ; J. Bartels and C. Ewerz, JHEP 9909, 026 (1999) [arXiv:hep-ph/9908454] ; J. Bartels and M. Wusthoff, Z. Phys. C66, 157 (1995); A. H. Mueller and B. Patel, Nucl. Phys. B425, 471 (1994) [arXiv:hep-ph/9403256]; J. Bartels, Z. Phys. C60, 471 (1993).

[16] M. A. Braun, Phys. Lett. B632 (2006) 297 [arXiv:hep-ph/0512057]; arXiv:hep-ph/0504002 ; Eur. Phys. J. C16, 337 (2000) [arXiv:hep-ph/0001268]; Phys. Lett. B 483 (2000) 115 [arXiv:hep-ph/0003004]; Eur. Phys. J. C 33 (2004) 113 [arXiv:hep-ph/0309293]; Eur. Phys. J. C6, 321 (1999) [arXiv:hep-ph/9706373]; M. A. Braun and G. P. Vacca, Eur. Phys. J. C6, 147 (1999) [arXiv:hep-ph/9711486].

[17] E. Iancu, A. H. Mueller and S. Munier, Phys. Lett. B 606 (2005) 342 [arXiv:hep-ph/0410018]; E. Brunet, B. Derrida, A. H. Mueller and S. Munier, arXiv:cond-mat/0603160; Phys. Rev. E73 (2006) 056126 [arXiv:cond-mat/0512021].

[18] R. Enberg, K. Golec-Biernat and S. Munier, Phys. Rev. D72 (2005) 074021 [arXiv:hep-ph/0505101]. S. Munier, arXiv:hep-ph/0608036.

[19] M. Kozlov, E. Levin and A. Prygarin, arXiv:hep-ph/0606260.

[20] M. Ciafaloni, D. Colferai, G.P. Salam and A.M. Stasto, Phys. Rev. D68 (2003) 114003; M. Ciafaloni, D. Colferai, G. P. Salam and A. M. Stasto, Phys. Lett. B541, 314 (2002) [arXiv:hep-ph/0204287]; Phys. Rev. D66, 054014 (2002) [arXiv:hep-ph/0204282]; V.S. Fadin and L.N. Lipatov, Phys. Lett. B429 (1998) 127

G. Camici and M. Ciafaloni,Phys. Lett. B430 (1998) 349.

[21] O.V. Kancheli, Sov. JETP Lett. 18, 274 (1973), in Caneschi, L. (ed.): "Regge theory of low-p(T) hadronic interactions', p. 312, North-Holland, 1989; Pisma Zh. Eksp. Teor. Fiz. 22 (1975) 491.

[22] A. H. Mueller, Nucl. Phys. B415, 373 (1994); ibid B437, 107 (1995).

[23] A. H. Mueller and B. Patel, Nucl. Phys. B425, 471 (1994); A. H. Mueller and G. P. Salam, Nucl. Phys. B475, 293 (1996), [arXiv:hep-ph/9605302]; G. P. Salam, Nucl. Phys. B461, 512 (1996); E. Iancu and A. H. Mueller, Nucl. Phys. A730 (2004) 460, 494, [arXiv:hep-ph/0308315],[arXiv:hep-ph/0309276]. 
[24] E. Levin and A. Prygarin, "The BFKL pomeron calculus in zero transverse dimension: Summation of the Pomeron loops and the generating functional for the multiparticle production processes," arXiv:hep-ph/0701178.

[25] E. Levin and K. Tuchin, Nucl. Phys. A 693 (2001) 787 [arXiv:hep-ph/0101275] ; Nucl. Phys. A 691 (2001) 779 [arXiv:hep-ph/0012167], Nucl. Phys. B 573 (2000) 833 [arXiv:hep-ph/9908317] .

[26] E. Levin and M. Lublinsky, Nucl. Phys. A730, 191 (2004) [arXiv:hep-ph/0308279].

[27] E. Levin and M. Lublinsky, Phys. Lett. B607, 131 (2005) [arXiv:hep-ph/0411121].

[28] S. Bondarenko, L. Motyka, A. H. Mueller, A. I. Shoshi and B. W. Xiao, "On the equivalence of Reggeon field theory in zero transverse dimensions and reaction-diffusion processes,", arXiv:hep-ph/0609213. P. Rembiesa and A. M. Stasto, Nucl. Phys. B725 (2005) 251 [arXiv:hep-ph/0503223]; A. I. Shoshi and B. W. Xiao, Phys. Rev. D 73, 094014 (2006) [arXiv:hep-ph/0512206]; Phys. Rev. D 75 (2007) 054002 [arXiv:hep-ph/0605282]; M. Kozlov and E. Levin, Nucl. Phys. A 779 (2006) 142 [ arXiv:hep-ph/0604039]; M. Kozlov, E. Levin, V. Khachatryan and J. Miller, "The BFKL pomeron calculus in zero transverse dimensions: Diffractive processes and survival probability for central diffractive production," arXiv:hep-ph/0610084.

[29] D. Amati, L. Caneschi and R. Jengo, Nucl. Phys. B 101 (1975) 397 V. Alessandrini, D. Amati and R. Jengo, Nucl. Phys. B 108 (1976) 425; R. Jengo, Nucl. Phys. B 108 (1976) 447; D. Amati, M. Le Bellac, G. Marchesini and M.Ciafaloni, Nucl. Phys. B112 (1976) 107; D. Amati, G. Marchesini, M.Ciafaloni and G. Parisi, Nucl. Phys. B114 (1976) 483; M. Ciafaloni, M. Le Bellac and G. C. Rossi, Nucl. Phys. B 130 (1977) 388.

[30] M. Kozlov, E. Levin, V. Khachatryan and J. Miller, arXiv:hep-ph/0610084.

[31] P. Grassberger and K. Sundermeyer, Phys. Lett. B77 (1978) 220.

[32] K. G. Boreskov, "Probabilistic model of Reggeon field theory," arXiv:hep-ph/0112325 and reference therein.

[33] V. A. Abramovsky, V. N. Gribov and O. V. Kancheli, Yad. Fiz. 18, 595 (1973) [Sov. J. Nucl. Phys. 18, 308 (1974)].

[34] Y. V. Kovchegov and E. Levin, Nucl. Phys. B 577 (2000) 221 [arXiv:hep-ph/9911523].

[35] Y. V. Kovchegov, Phys. Rev. D 72 (2005) 094009 [arXiv:hep-ph/0508276].

[36] M. Kozlov and E. Levin, Nucl. Phys. A739 (2004) 291 [arXiv:hep-ph/0401118].

[37] E. Levin, Nucl. Phys. A763, 140 (2005), arXiv:hep-ph/0502243.

[38] M. Kozlov and E. Levin, "Solution for the BFKL pomeron calculus in zero transverse dimensions," arXiv:hep-ph/0604039.

[39] S. Bondarenko, L. Motyka, A. H. Mueller, A. I. Shoshi and B. W. Xiao, arXiv:hep-ph/0609213.

[40] I. Gradstein and I. Ryzhik, " Tables of Series, Products, and Integrals", Verlag MIR, Moskau,1981.

[41] M. Abramowitz and I. Stegun, "Handbook of Mathematical Function", Dover Publications, Inc., New York, 1972.

[42] L. N. Lipatov, Phys. Rept. 286, 131 (1997) [arXiv:hep-ph/9610276]; Sov. Phys. JETP 63, 904 (1986) and references therein.

[43] H. Navelet and R. Peschanski, Nucl. Phys. B634, 291 (2002) [arXiv:hep-ph/0201285]; Phys. Rev. Lett. 82, 137 (1999), [arXiv:hep-ph/9809474]; Nucl. Phys. B507, 353 (1997) [arXiv:hep-ph/9703238].

[44] Y. Hatta and A. H. Mueller, “Correlation of small-x gluons in impact parameter space,” arXiv:hep-ph/0702023.

[45] A. H. Mueller and A. I. Shoshi, Nucl. Phys. B692 (2004) 175 [arXiv:hep-ph/0402193].

[46] J. Bartels and E. Levin, Nucl. Phys. B387 (1992) 617.

[47] A. H. Mueller and D. N. Triantafyllopoulos, Nucl. Phys. B640 (2002) 331 [arXiv:hep-ph/0205167]; D. N. Triantafyllopoulos, Nucl. Phys. B 648 (2003) 293 [arXiv:hep-ph/0209121].

[48] S. Munier and R. Peschanski, Phys. Rev. D70 (2004) 077503; D69 (2004) 034008 [arXiv:hep-ph/0310357]; Phys. Rev. Lett. 91 (2003) 232001 [arXiv:hep-ph/0309177]. 
[49] A. H. Mueller, Nucl. Phys. B558 (1999) 285, [arXiv:hep-ph/9904404].

[50] M. Kozlov, E. Levin and A. Prygarin, "The BFKL pomeron calculus in the dipole approach," arXiv:0704.2124 [hep-ph]; S. Bondarenko, "Langevin equation in effective theory of interacting QCD pomerons in the limit of large $N_{c}$," arXiv:hep-ph/0703045.

[51] http://mathworld.wolfram.com/ConfluentHypergeometricLimitFunction.html

[52] J. Bartels, M. A. Braun, D. Colferai and G. P. Vacca, Eur. Phys. J. C 20, 323 (2001) [arXiv:hep-ph/0102221].

[53] K. J. Golec-Biernat and M. Wusthoff, Phys. Rev. D 59 (1999) 014017 [arXiv:hep-ph/9807513] ; Phys. Rev. D 60 (1999) 114023 [arXiv:hep-ph/9903358] ; E. Gotsman, E. Levin, M. Lublinsky, U. Maor, E. Naftali and K. Tuchin, J. Phys. G 27 (2001) 2297 [arXiv:hep-ph/0010198] ; H. Kowalski and D. Teaney, Phys. Rev. D 68 (2003) 114005 [arXiv:hep-ph/0304189] ; J. Bartels, K. J. Golec-Biernat and H. Kowalski, Phys. Rev. D 66 (2002) 014001 [arXiv:hep-ph/0203258].

[54] E. Levin, Nucl. Phys. B 453 (1995) 303 [arXiv:hep-ph/9412345] ; M. A. Braun, Phys. Lett. B 348 (1995) 190 [arXiv:hep-ph/9408261].

[55] Y. V. Kovchegov and H. Weigert, Nucl. Phys. A 789 (2007) 260 [arXiv:hep-ph/0612071] ; Nucl. Phys. A 784 (2007) 188 [arXiv:hep-ph/0609090] ; E. Gardi, J. Kuokkanen, K. Rummukainen and H. Weigert, Nucl. Phys. A 784 (2007) 282 [arXiv:hep-ph/0609087] ; I. Balitsky, Phys. Rev. D 75 (2007) 014001 [arXiv:hep-ph/0609105]. 